\title{
$W^{a}$ Ve phenomena

\section{Dual variational methods for a nonlinear Helmholtz system}

Rainer Mandel, Dominic Scheider

CRC Preprint 2017/25, October 2017

\section{KARLSRUHE INSTITUTE OF TECHNOLOGY}

\section{CRC 1173}

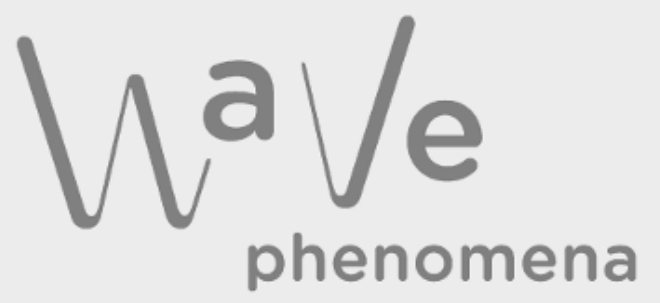




\section{Participating universities}

EBERHARD KARLS

UNIVERSITATT TUBINGEN

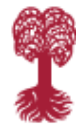

Funded by

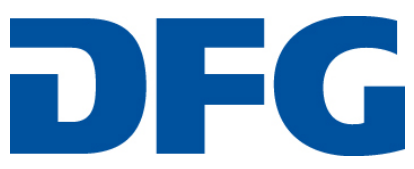

ISSN 2365-662X 


\title{
DUAL VARIATIONAL METHODS FOR A NONLINEAR HELMHOLTZ SYSTEM
}

\author{
RAINER MANDEL AND DOMINIC SCHEIDER
}

Abstract. This paper considers a pair of coupled nonlinear Helmholtz equations

$$
\left\{\begin{array}{l}
-\Delta u-\mu u=a(x)\left(|u|^{\frac{p}{2}}+b(x)|v|^{\frac{p}{2}}\right)|u|^{\frac{p}{2}-2} u, \\
-\Delta v-\nu v=a(x)\left(|v|^{\frac{p}{2}}+b(x)|u|^{\frac{p}{2}}\right)|v|^{\frac{p}{2}-2} v
\end{array}\right.
$$

on $\mathbb{R}^{N}$ where $\frac{2(N+1)}{N-1}<p<2^{*}$. The existence of nontrivial strong solutions in $W^{2, p}\left(\mathbb{R}^{N}\right)$ is established using dual variational methods. The focus lies on necessary and sufficient conditions on the parameters deciding whether or not both components of such solutions are nontrivial.

\section{Introduction And Main Results}

In this paper we study a system of two coupled nonlinear Helmholtz equations as it arises in models of nonlinear optics. More specifically, we intend to find a pair of fully nontrivial, real-valued and strong solutions $(u, v) \in W^{2, p}\left(\mathbb{R}^{N}\right) \times W^{2, p}\left(\mathbb{R}^{N}\right), N \geq 2$, of

$$
\begin{cases}-\Delta u-\mu u=a(x)\left(|u|^{\frac{p}{2}}+b(x)|v|^{\frac{p}{2}}\right)|u|^{\frac{p}{2}-2} u & \text { on } \mathbb{R}^{N}, \\ -\Delta v-\nu v=a(x)\left(|v|^{\frac{p}{2}}+b(x)|u|^{\frac{p}{2}}\right)|v|^{\frac{p}{2}-2} v & \text { on } \mathbb{R}^{N}, \\ u, v \in L^{p}\left(\mathbb{R}^{N}\right) & \end{cases}
$$

with $\mu, \nu>0, \frac{2(N+1)}{N-1}<p<2^{*}$ and nonnegative, $\mathbb{Z}^{N}$-periodic coefficients $a, b \in L^{\infty}\left(\mathbb{R}^{N}\right)$. Here $2^{*}$ denotes the critical Sobolev exponent, $2^{*}=\frac{2 N}{N-2}$ for $N \geq 3,2^{*}=\infty$ for $N=2$. A solution $(u, v)$ of (11) is said to be semitrivial if either $u=0$ or $v=0$ and fully nontrivial if both $u \neq 0$ and $v \neq 0$. Our aim is to find necessary and sufficient conditions for the existence of fully nontrivial solutions of (1).

To our knowledge, systems of Helmholtz equations have not been discussed in the literature so far in contrast to the Schrödinger case where $\mu, \nu<0$ in (11); for a comparison with our results, we refer to the end of this introduction. Likewise, there is much literature on nonlinear Schrödinger equations of the form

$$
-\Delta w+\lambda w=Q(x)|w|^{p-2} w \quad \text { on } \mathbb{R}^{N}
$$

with some $\lambda>0$; for instance, Szulkin and Weth prove the existence of ground state solutions in the Sobolev space $H^{1}\left(\mathbb{R}^{N}\right)$ in Theorem 1.1 of [19] by constraint minimization on the

Date: October 13, 2017.

2010 Mathematics Subject Classification. Primary: 35J50, Secondary: 35J05.

Key words and phrases. Nonlinear Helmholtz sytem, dual variational methods. 
associated Nehari set. In contrast, the corresponding Helmholtz problem

$$
-\Delta w-\lambda w=Q(x)|w|^{p-2} w \quad \text { on } \mathbb{R}^{N}
$$

has only been discussed during the past five years. Since $\lambda>0$ belongs to the essential spectrum of $-\Delta$, the Helmholtz case requires different concepts in order to handle oscillating solutions with slow decay which, in general, are not elements of $H^{1}\left(\mathbb{R}^{N}\right)$. In the radial case, such oscillation and decay properties are studied in [16]. Evéquoz and Weth discuss the case of compactly supported $Q$ and $2<p<2^{*}$ in [6, 8]. In [6], they study an exterior problem where the nonlinearity vanishes and knowledge about the far-field expansion of solutions is available. The remaining problem on a bounded domain is solved by variational techniques. The approach in [8] uses Leray-Schauder continuation with respect to the parameter $\lambda$ in order to find solutions of (2). We will follow the ideas of Evéquoz and Weth presented in [3, 7 . They introduce a dual variational approach, transforming the Helmholtz equation (21) into

$$
|\bar{w}|^{p^{\prime}-2} \bar{w}=Q(x)^{\frac{1}{p}}(-\Delta-\lambda)^{-1}\left[Q(x)^{\frac{1}{p}} \bar{w}\right] \quad \text { where } \bar{w}(x)=Q(x)^{\frac{1}{p^{\prime}}}|w(x)|^{p-2} w(x) .
$$

Here, the resolvent-type operator $(-\Delta-\lambda)^{-1}$ is obtained by the Limiting Absorption Principle of Gutiérrez, see the pretext of Theorem 6 in [10]. The resulting dual equation in $L^{p^{\prime}}\left(\mathbb{R}^{N}\right)$ is variational; using the Mountain Pass Theorem, the authors prove the existence of a ground state $\bar{w} \in L^{p^{\prime}}\left(\mathbb{R}^{N}\right)$ of the dual problem, which yields a strong solution $w \in W^{2, q}\left(\mathbb{R}^{N}\right) \cap$ $C^{1, \alpha}\left(\mathbb{R}^{N}\right), q \in[p, \infty)$ and $\alpha \in(0,1)$, of the Helmholtz equation (2). Here $\frac{2(N+1)}{N-1}<p<2^{*}$ and $Q$ is assumed to be positive and either periodic or decaying at infinity. In the latter case, it is shown that infinitely many solutions exist; for periodic $Q$, Evéquoz proves the corresponding statement in [5]. He also shows that the dual problem possesses a gound state if $Q$ is assumed to be the sum of a periodic and a decaying term. Evéquoz further generalizes these results in [2]; for instance, it is shown that the dual variational techniques apply for any $p \in\left(2,2^{*}\right)$ if $Q$ satisfies suitable integrability conditions. In [9], Evéquoz and Yeşil prove the existence of a dual ground state in the critical case $p=2^{*}$ for $N \geq 4$ and the non-existence for $N=3$ where, again, $Q$ is assumed to be the sum of a decaying and a periodic term. For continuous, nonnegative $Q$ and $\frac{2(N+1)}{N-1}<p<2^{*}$, Evéquoz proves existence, concentration and multiplicity of ground states of the dual problem in the high-frequency limit $\lambda \nearrow \infty$ in [4] based on a comparison of energies with a suitable limit problem.

We will show that, under suitable assumptions on the coefficients $a$ and $b$, the dual variational approach and the existence results by Evéquoz and Weth in [3, 7] extend to the case of the system (11). To this end, we will introduce a dual formulation for the system (11) of the form

$$
\begin{cases}\partial_{\bar{s}} h(x, \bar{u}, \bar{v})=\Psi_{\mu} * \bar{u} & \text { on } \mathbb{R}^{N}, \\ \partial_{\bar{t}} h(x, \bar{u}, \bar{v})=\Psi_{\nu} * \bar{v} & \text { on } \mathbb{R}^{N}, \\ \bar{u}, \bar{v} \in L^{p^{\prime}}\left(\mathbb{R}^{N}\right) . & \end{cases}
$$


In the following section, the role of the convolution kernels $\Psi_{\mu}, \Psi_{\nu}$, see also equations (11), (45) of [7], will be explained. So will be the transformation involving $\bar{u}, \bar{v} \in L^{p^{\prime}}\left(\mathbb{R}^{N}\right)$,

$$
\begin{aligned}
& \bar{u}(x):=a(x)\left(|u(x)|^{\frac{p}{2}}+b(x)|v(x)|^{\frac{p}{2}}\right)|u(x)|^{\frac{p}{2}-2} u(x), \\
& \bar{v}(x):=a(x)\left(|v(x)|^{\frac{p}{2}}+b(x)|u(x)|^{\frac{p}{2}}\right)|v(x)|^{\frac{p}{2}-2} v(x)
\end{aligned}
$$

and a suitable function $h: \mathbb{R}^{N} \times \mathbb{R} \times \mathbb{R} \rightarrow \mathbb{R}$, cf. Proposition 1 below. Notice that we use the notation $\bar{u}, \bar{v} \in L^{p^{\prime}}\left(\mathbb{R}^{N}\right)$ in place of $u, v \in L^{p}\left(\mathbb{R}^{N}\right)$ whenever we are working in the dual setting; it does not denote complex conjugation, which does not occur in this paper.

The dual system (3) is variational; we introduce the corresponding energy functional

$$
\begin{aligned}
& J_{\mu \nu}: \quad L^{p^{\prime}}\left(\mathbb{R}^{N}\right) \times L^{p^{\prime}}\left(\mathbb{R}^{N}\right) \rightarrow \mathbb{R} \\
& J_{\mu \nu}(\bar{u}, \bar{v}):=\int_{\mathbb{R}^{N}} h(x, \bar{u}, \bar{v}) \mathrm{d} x-\frac{1}{2} \int_{\mathbb{R}^{N}} \bar{u} \Psi_{\mu} * \bar{u}+\bar{v} \Psi_{\nu} * \bar{v} \mathrm{~d} x
\end{aligned}
$$

with mountain pass level

$$
\begin{aligned}
& c_{\mu \nu}:=\inf _{\gamma \in \Gamma \mu \nu} \sup _{0 \leq t \leq 1} J_{\mu \nu}(\gamma(t)) \\
& \text { where } \Gamma_{\mu \nu}:=\left\{\gamma \in C\left([0,1], L^{p^{\prime}}\left(\mathbb{R}^{N}\right) \times L^{p^{\prime}}\left(\mathbb{R}^{N}\right)\right): \gamma(0)=0, J_{\mu \nu}(\gamma(1))<0\right\} .
\end{aligned}
$$

For the definition of $h$, we refer to Proposition 1. The main results will be proved under the following assumptions:

$$
\begin{gathered}
N \geq 2, \quad \mu, \nu>0, \quad \frac{2(N+1)}{N-1}<p<2^{*}, \\
a, b \in L^{\infty}\left(\mathbb{R}^{N}\right) \text { are }[0,1]^{N} \text {-periodic with } 0 \leq b(x) \leq p-1, a(x) \geq a_{0}>0 .
\end{gathered}
$$

We denote by $a_{-}, b_{-}$the (essential) infimum and by $a_{+}, b_{+}$the (essential) supremum of the functions $a$ and $b$, respectively.

Theorem 1 (Existence Theorem). Assuming (7), there exists a nontrivial critical point $(\bar{u}, \bar{v}) \in L^{p^{\prime}}\left(\mathbb{R}^{N}\right) \times L^{p^{\prime}}\left(\mathbb{R}^{N}\right)$ of the functional $J_{\mu \nu}$ on the mountain pass level $c_{\mu \nu}>0$ and $(u, v):=\nabla_{\bar{s}, \bar{t}} h(\cdot, \bar{u}, \bar{v})$ is a strong solution of (1) with $u, v \in W^{2, q}\left(\mathbb{R}^{N}\right) \cap C^{1, \alpha}\left(\mathbb{R}^{N}\right)$ for all $q \in[p, \infty)$ and $\alpha \in(0,1)$.

Remark 1. Consider the scalar functional $I_{\mu}: L^{p^{\prime}}\left(\mathbb{R}^{N}\right) \rightarrow \mathbb{R}$ given by $I_{\mu}(\bar{u}):=J_{\mu \nu}(\bar{u}, 0)$. Lemma 1 (d) below provides the formula

$$
I_{\mu}(\bar{u})=\frac{1}{p^{\prime}} \int_{\mathbb{R}^{N}} a(x)^{1-p^{\prime}}|\bar{u}|^{p^{\prime}} \mathrm{d} x-\frac{1}{2} \int_{\mathbb{R}^{N}} \bar{u} \Psi_{\mu} * \bar{u} \mathrm{~d} x .
$$

The results by Evéquoz and Weth in [3, 7] yield a critical point $\bar{u}$ of $I_{\mu}$ at the scalar mountain pass level $c_{\mu}$ and a corresponding solution $u=a^{1-p^{\prime}}|\bar{u}|^{p^{\prime}-2} \bar{u} \in W^{2, q}\left(\mathbb{R}^{N}\right) \cap C^{1, \alpha}\left(\mathbb{R}^{N}\right), q \in$ $[p, \infty)$ and $\alpha \in(0,1)$, of the scalar Helmholtz equation

$$
-\Delta u-\mu u=a(x)|u|^{p-2} u \quad \text { on } \mathbb{R}^{N} \text {. }
$$


Any critical point of the functional $J_{\mu \nu}$ on the level $c_{\mu \nu}$ will henceforth be referred to as a dual ground state of $J_{\mu \nu}$. Notice that Theorem 1 yields the existence of a nontrivial dual ground state $(\bar{u}, \bar{v})$ of (3) ; it does not exclude the semitrivial case where either $\bar{u}=0$ or $\bar{v}=0$. Such a semitrivial dual ground state corresponds to a solution of the scalar problem; thus we now discuss under which conditions we find fully nontrivial dual ground states of the system (3). This amounts to statements about the occurrence of fully nontrivial solutions of (1). Indeed, for pairs $(\bar{u}, \bar{v}) \in L^{p^{\prime}}\left(\mathbb{R}^{N}\right) \times L^{p^{\prime}}\left(\mathbb{R}^{N}\right)$ and $(u, v) \in L^{p}\left(\mathbb{R}^{N}\right) \times L^{p}\left(\mathbb{R}^{N}\right)$ satisfying (44), a short calculation shows that $(u, v)$ is semitrivial (resp. fully nontrivial) if and only if $(\bar{u}, \bar{v})$ is semitrivial (resp. fully nontrivial).

Theorem 2. Assume conditions (17) to hold.

(a) If $2<p<4$ and $b_{-}>0$, then every dual ground state of the functional $J_{\mu \nu}$ is fully nontrivial.

(b) If $\quad p \geq 4$ and $b_{-}>\frac{a_{+}}{a_{-}} 2^{\frac{p-2}{2}}-1$, then there exists $\delta>0$ with the property that, for $\mu, \nu>0$ with $\left|\sqrt{\frac{\mu}{\nu}}-1\right|<\delta$, every dual ground state of $J_{\mu \nu}$ is fully nontrivial.

Theorem 3. Assume (7) as well as

$$
p \geq 4 \quad \text { and } \quad 0 \leq b_{+}<2^{\frac{p-2}{2}}-1 .
$$

Then every dual ground state of the functional $J_{\mu \nu}$ is semitrivial.

In the special case of constant coefficients $a, b$ and $\mu=\nu$ we provide a full characterization of the parameter ranges where semitrivial and fully nontrivial dual ground state solutions occur:

Corollary 1. Assume that conditions (7) hold with constant coefficients $a(x) \equiv a>0$ and $b(x) \equiv b \in[0, p-1]$. Then we have the following:

(a) $J_{\mu \mu}$ attains the level $c_{\mu \mu}$ in a fully nontrivial dual ground state if and only if

$$
2<p<4 \text { and } b>0 \quad \text { or } \quad p \geq 4 \text { and } b \geq 2^{\frac{p-2}{2}}-1 \text {. }
$$

(b) $J_{\mu \mu}$ attains the level $c_{\mu \mu}$ in a semitrivial dual ground state if and only if

$$
2<p<4 \text { and } b=0 \quad \text { or } \quad p \geq 4 \text { and } 0 \leq b \leq 2^{\frac{p-2}{2}}-1 .
$$

The proofs of these results will be given in section 5 . They essentially consist of a comparison of the energy levels $c_{\mu \nu}$ and $\min \left\{c_{\mu}, c_{\nu}\right\}$, cf. Lemma 5 in section 4 .

Remark 2. If (77) holds and $p>8$, we have $2^{\frac{p-2}{2}}-1>p-1$ and thus by Theorem 3, only semitrivial dual ground states occur.

Thanks to a hint by Evéquoz (yet unpublished), one can weaken assumptions (7) imposing radial symmetry.

Remark 3. If we consider spaces of radial functions and constant coefficients $a, b$, all above Theorems hold under the weaker assumption $\frac{2 N}{N-1}<p<2^{*}$ instead of $\frac{2(N+1)}{N-1}<p<2^{*}$. 
Indeed, for the construction of a continuous resolvent $(-\Delta-\lambda)^{-1}: L^{p^{\prime}}\left(\mathbb{R}^{N}, \mathbb{C}\right) \rightarrow L^{p}\left(\mathbb{R}^{N}, \mathbb{C}\right)$, Evéquoz and Weth refer to a result by Gutiérrez, Theorem 6 in [10]. A crucial step in its proof, to be found on p. 19 of [10], is the estimate

$$
\int_{\mathbb{S}^{N-1}}|\mathcal{F} g(r \omega)|^{2} \mathrm{~d} \sigma(\omega) \leq C r^{-\frac{2 N}{p^{\prime}}}\|g\|_{L^{p}\left(\mathbb{R}^{N}\right)}^{2} \quad \text { for } 1 \leq p \leq \frac{2(N+1)}{N+3}
$$

where $g: \mathbb{R} \rightarrow \mathbb{C}$ is a Schwartz function and $\mathrm{d} \sigma$ denotes the surface measure on the sphere $\mathbb{S}^{N-1} \subseteq \mathbb{R}^{N}$. It is a consequence of the Stein-Tomas Theorem, see p. 375 and p. 414 in [18] for $N \geq 3$ and $N=2$, respectively. In the radial case, however, Evéquoz is able to show that $\|\mathcal{F} h\|_{L_{\mathrm{d} \sigma}^{\infty}\left(\mathbb{S}^{N-1}, \mathbb{C}\right)} \leq C_{p}\|h\|_{L^{p}\left(\mathbb{R}^{N}, \mathbb{C}\right)}$ for all radial Schwartz functions $h: \mathbb{R} \rightarrow \mathbb{C}$ and $1 \leq p<\frac{2 N}{N+1}$. Replacing estimate (10) by

$$
\int_{\mathbb{S}^{N-1}}|\mathcal{F} g(r \omega)|^{2} \mathrm{~d} \sigma(\omega) \leq \tilde{C} r^{-\frac{2 N}{p^{\prime}}}\|g\|_{L^{p}\left(\mathbb{R}^{N}\right)}^{2} \quad \text { for } 1 \leq p \leq \frac{2 N}{N+1}
$$

for radial Schwartz functions $g: \mathbb{R} \rightarrow \mathbb{C}$, he thus concludes that, in the radial case, the Helmholtz resolvent $(-\Delta-\lambda)^{-1}: L_{\mathrm{rad}}^{p^{\prime}}\left(\mathbb{R}^{N}, \mathbb{C}\right) \rightarrow L_{\mathrm{rad}}^{p}\left(\mathbb{R}^{N}, \mathbb{C}\right)$ is continuous for $\frac{2 N}{N-1}<p<2^{*}$.

Finally, let us briefly compare our results concerning the occurrence of fully nontrivial ground state solutions to those available in the case of a Schrödinger system, i.e. $\mu, \nu<0$ in (11). We assume $2<p<2^{*}$ and constant coupling $b(x) \equiv \beta \neq 0$; with $\omega:=\sqrt{\frac{\nu}{\mu}}$, we discuss

$$
\begin{cases}-\Delta u+\quad u=\left(|u|^{\frac{p}{2}}+\beta|v|^{\frac{p}{2}}\right)|u|^{\frac{p}{2}-2} u & \text { on } \mathbb{R}^{N} \\ -\Delta v+\omega^{2} v=\left(|v|^{\frac{p}{2}}+\beta|u|^{\frac{p}{2}}\right)|v|^{\frac{p}{2}-2} v & \text { on } \mathbb{R}^{N} \\ u, v \in H^{1}\left(\mathbb{R}^{N}\right) & \end{cases}
$$

Sharp characterizations of the occurrence of fully nontrivial ground state solutions have been provided by the first author in [15] for the cooperative case $\beta>0$, following pioneering work by Ambrosetti and Colorado [1], Maia, Montefusco and Pellacci [12] and others. In contrast to the Helmholtz case, the parameter $p$ can be chosen from the full superlinear and subcritical range $2<p<2^{*}$ whereas in the Helmholtz case, we use mapping properties of the resolvent available only for $\frac{2(N+1)}{N-1}<p<2^{*}$. Moreover, in order to obtain a suitable dual formulation, our discussion for the Helmholtz system only covers the range $0 \leq \beta \leq p-1$; in particular, we only study cooperative systems. In the Schrödinger case, results for the repulsive case $\beta<0$ are available as well, see for instance [13] and the references therein. Notice that in the special case $\omega=1$ and $\mu=\nu$ in (3), the ranges for $p$ and $\beta$ in Theorem 1 and Remark 1(a) of [15] for the Schrödinger case agree with those from Corollary 1 above for the Helmholtz case. Finally, in the situation of Theorem 2 (b) of the Helmholtz case, the question remains open whether there are threshold values for the existence and non-existence of fully nontrivial ground state solutions such as in Theorem 1 in [15]. 


\section{The DUAl Formulation, CONVEXity AND The LEgEndRE TRANSForm}

In this section, we intend to explain and justify the transition from the nonlinear Helmholtz system (11) to (3). Let us first note that the system (1) can be written in the form

$$
\begin{cases}-\Delta u-\mu u=\partial_{s} f(x, u, v) & \text { on } \mathbb{R}^{N} \\ -\Delta v-\nu v=\partial_{t} f(x, u, v) & \text { on } \mathbb{R}^{N} \\ u, v \in L^{p}\left(\mathbb{R}^{N}\right) & \end{cases}
$$

where

$$
f: \mathbb{R}^{N} \times \mathbb{R} \times \mathbb{R} \rightarrow \mathbb{R}, \quad f(x, s, t)=\frac{a(x)}{p}\left(|s|^{p}+2 b(x)|s|^{\frac{p}{2}}|t|^{\frac{p}{2}}+|t|^{p}\right) .
$$

The transformation (4) then reads $(\bar{u}(x), \bar{v}(x))=\nabla_{s, t} f(x, u(x), v(x))$ for $x \in \mathbb{R}^{N}$.

We first provide the definition and the most important properties of the convolution terms in (3), referring to section 2 of [7] and the beginning of section 4 in [3] for more details. We denote by $\mathcal{F}$ the Fourier transform on the Schwartz space $\mathcal{S}\left(\mathbb{R}^{N}, \mathbb{C}\right)$. For $\lambda>0$ and $\varepsilon>0$, the operator $-\Delta-(\lambda+\mathrm{i} \varepsilon): \mathcal{S}\left(\mathbb{R}^{N}, \mathbb{C}\right) \rightarrow \mathcal{S}\left(\mathbb{R}^{N}, \mathbb{C}\right)$ possesses a resolvent given by

$$
\mathcal{R}_{\varepsilon}^{\lambda}: \mathcal{S}\left(\mathbb{R}^{N}, \mathbb{C}\right) \rightarrow \mathcal{S}\left(\mathbb{R}^{N}, \mathbb{C}\right), g \mapsto \mathcal{F}^{-1}\left(\left(|\cdot|^{2}-(\lambda+\mathrm{i} \varepsilon)\right)^{-1} \cdot \mathcal{F}(g)\right) .
$$

By the Limiting Absorption Principle of Gutiérrez, cf. the pretext of Theorem 6 in [10], it is possible to pass to the limit $\varepsilon \searrow 0$, which yields an operator

$$
\mathcal{R}^{\lambda}: \mathcal{S}\left(\mathbb{R}^{N}, \mathbb{C}\right) \rightarrow \mathcal{S}^{\prime}\left(\mathbb{R}^{N}, \mathbb{C}\right), \quad \mathcal{R}^{\lambda} g[w]=\int_{\mathbb{R}^{N}}\left(\Phi_{\lambda} * g\right) \cdot w \mathrm{~d} x
$$

where the kernel $\Phi_{\lambda}: \mathbb{R}^{N} \rightarrow \mathbb{C}$ is given by

$$
\Phi_{\lambda}(x)=\lambda^{\frac{N-2}{2}} \Phi_{1}(\sqrt{\lambda} x)=\frac{\mathrm{i}}{4}\left(\frac{\lambda}{4 \pi^{2}|x|^{2}}\right)^{\frac{N-2}{4}} H_{\frac{N-2}{2}}^{(1)}(|\sqrt{\lambda} x|) \quad\left(x \in \mathbb{R}^{N}, \lambda>0\right),
$$

cf. the pretext of equation (6) in [3] for $N=2$ and equation (11) in [7] for $N \geq 3$. It can be shown that there exists a continuous extension

$$
\mathcal{R}^{\lambda}: L^{p^{\prime}}\left(\mathbb{R}^{N}, \mathbb{C}\right) \rightarrow L^{p}\left(\mathbb{R}^{N}, \mathbb{C}\right), \quad \bar{w} \mapsto \Phi_{\lambda} * \bar{w} \quad \text { for } \frac{2(N+1)}{N-1}<p<2^{*},
$$

cf. Theorem 2.1 both in [3] and in [7]. Proposition A.1 in [7] now states that, for $f \in$ $L^{p^{\prime}}\left(\mathbb{R}^{N}, \mathbb{C}\right)$, the function $u:=\mathcal{R}^{\lambda} f \in L^{p}\left(\mathbb{R}^{N}, \mathbb{C}\right)$ is in fact a strong solution of the linear Helmholtz equation $-\Delta u-\lambda u=f$ on $\mathbb{R}^{N}$. In this sense, the operator $\mathcal{R}^{\lambda}$ can be interpreted as a resolvent operator for the linear Helmholtz equation.

Being interested in real-valued solutions, we introduce $\Psi_{\lambda}:=\Re \Phi_{\lambda}$ and, from now on, consider spaces of real-valued functions. Then we have continuity of $\Psi_{\mu} *[\cdot], \Psi_{\nu} *[\cdot]: L^{p^{\prime}}\left(\mathbb{R}^{N}\right) \rightarrow$ 
$L^{p}\left(\mathbb{R}^{N}\right)$ and we can find solutions of the system (1) by solving

$$
\begin{cases}u=\Psi_{\mu} * \partial_{s} f(x, u, v) & \text { on } \mathbb{R}^{N}, \\ v=\Psi_{\nu} * \partial_{t} f(x, u, v) & \text { on } \mathbb{R}^{N}, \\ u, v \in L^{p}\left(\mathbb{R}^{N}\right) . & \end{cases}
$$

As mentioned earlier, we aim to reformulate the system (14) by, roughly speaking, replacing the functions $u, v \in L^{p}\left(\mathbb{R}^{N}\right)$ by a corresponding pair

$$
\bar{u}, \bar{v} \in L^{p^{\prime}}\left(\mathbb{R}^{N}\right) \quad \text { via } \quad \bar{u}:=\partial_{s} f(\cdot, u, v), \bar{v}:=\partial_{t} f(\cdot, u, v),
$$

see also (4), such that the convolutions occur in the linear part of the equations. We will see in Proposition 1 that, under suitable assumptions on the coefficients $a$ and $b$, this transformation is invertible and preserves the variational structure in the sense that

$$
u=\partial_{\bar{s}} h(\cdot, \bar{u}, \bar{v}), \quad v=\partial_{\bar{t}} h(\cdot, \bar{u}, \bar{v})
$$

with a suitable function $h: \mathbb{R}^{N} \times \mathbb{R} \times \mathbb{R} \rightarrow \mathbb{R}$, which then finally provides a one-to-one correspondence between solutions of the systems (14) and (3). It turns out that we have to choose $h(x, \cdot, \cdot)$ to be the Legendre transform of $f(x, \cdot, \cdot)$ for every fixed $x \in \mathbb{R}^{N}$. We remark that, in the case of a single nonlinear Helmholtz equation (2), $-\Delta w-\lambda w=Q(x)|w|^{p-2} w$ on $\mathbb{R}^{N}$, the associated change of variables can be done explicitly, $\bar{w}(x):=Q(x)|w(x)|^{p-2} w(x)$ and hence $w(x)=Q(x)^{1-p^{\prime}}|\bar{w}(x)|^{p^{\prime}-2} \bar{w}(x)$. Notice that the treatment of the term $Q$ slightly differs from that in [3,7].

By Theorems 26.5 and 26.6 in [17], we have the following general result of convex analysis:

Theorem 4. Let $F: \mathbb{R}^{2} \rightarrow \mathbb{R}$ be differentiable, strictly convex and co-finite. Then $\nabla F:$ $\mathbb{R}^{2} \rightarrow \mathbb{R}^{2}$ is a homeomorphism, and the Legendre transform of $F$,

$$
H: \mathbb{R}^{2} \rightarrow \mathbb{R}, \quad H(\bar{s}, \bar{t}):=\sup _{(s, t) \in \mathbb{R}^{2}}(s \bar{s}+t \bar{t}-F(s, t))
$$

is well-defined, differentiable, strictly convex, co-finite and satisfies $\nabla H=(\nabla F)^{-1}$.

Let us remark that, for a convex function $F: \mathbb{R}^{2} \rightarrow \mathbb{R}$, co-finiteness is characterized by

$$
\lim _{\lambda \rightarrow \infty} \frac{F(\lambda s, \lambda t)}{\lambda}=\infty \text { for all }(s, t) \neq(0,0),
$$

cf. the equation before Theorem 26.6 in [17]. We check that, under the assumptions (77) and for fixed $x \in \mathbb{R}^{N}$, Theorem 4 applies to the function

$$
f(x, \cdot, \cdot): \mathbb{R}^{2} \rightarrow \mathbb{R}, \quad f(x, s, t)=\frac{a(x)}{p}\left(|s|^{p}+2 b(x)|s|^{\frac{p}{2}}|t|^{\frac{p}{2}}+|t|^{p}\right)
$$

so that a dual variational formulation for (1) is available and given by (3).

Proposition 1. Let $p>2$ and $x \in \mathbb{R}^{N}$ with $a(x)>0$ and $0 \leq b(x) \leq p-1$. Then the function $f(x, \cdot, \cdot)$ is differentiable, strictly convex and co-finite. Hence, its Legendre transform $h(x, \cdot, \cdot)$ is well-defined, differentiable, strictly convex, co-finite and satisfies $\nabla_{\bar{s}, \bar{t}} h(x, \cdot, \cdot)=\left(\nabla_{s, t} f(x, \cdot, \cdot)\right)^{-1}$. 
The proof is given in the appendix. Let us emphasize that it is only this existence result which requires the assumption $0 \leq b(x) \leq p-1$ in (7). In some cases, the Legendre transform $h$ can be calculated explicitly. For instance, in the case $b(x) \equiv 1$, we have for all $x \in \mathbb{R}^{N}$ and $s, t \in \mathbb{R}$

$$
\begin{array}{ll}
f(x, s, t)=\frac{a(x)}{p}\left(|s|^{\frac{p}{2}}+|t|^{\frac{p}{2}}\right)^{2}, & \nabla_{s, t} f(x, s, t)=a(x)\left(|s|^{\frac{p}{2}}+|t|^{\frac{p}{2}}\right)\left(\begin{array}{c}
|s|^{\frac{p}{2}-2} s \\
|t|^{\frac{p}{2}-2} t
\end{array}\right), \\
h(x, \bar{s}, \bar{t})=\frac{a(x)^{1-p^{\prime}}}{p^{\prime}}\left(|\bar{s}|^{\frac{p}{p-2}}+|\bar{t}|^{\frac{p}{p-2}}\right)^{\frac{p-2}{p-1}}, & \nabla_{\bar{s}, \bar{t}} h(x, \bar{s}, \bar{t})=\left(a(x)\left(|\bar{s}|^{\frac{p}{p-2}}+|\bar{t}|^{\frac{p}{p-2}}\right)\right)^{1-p^{\prime}}\left(\begin{array}{l}
|\bar{s}|^{\frac{4-p}{p-2}} \bar{s} \\
|\bar{t}|^{\frac{4-p}{p-2}} \bar{t}
\end{array}\right) .
\end{array}
$$

In the following sections we will need some properties of $h(x, \cdot, \cdot)$ which are listed next and will be proved in the appendix.

Lemma 1. Let $p>2$ and $x \in \mathbb{R}^{N}$ with $a(x)>0,0 \leq b(x) \leq p-1$. Then, for $\bar{s}, \bar{t} \in \mathbb{R}$,

$$
\begin{aligned}
& \text { (a) } h(x, \bar{s}, \bar{t})=\frac{a(x)^{1-p^{\prime}}}{p^{\prime}}\left[\sup _{\sigma>0} \frac{|\bar{s}|+\sigma|\bar{t}|}{\left(1+2 b(x) \sigma^{\frac{p}{2}}+\sigma^{p}\right)^{\frac{1}{p}}}\right]^{p^{\prime}}, \\
& \text { (b) } h(x, \bar{s}, \bar{t})=h(x, \bar{t}, \bar{s})=h(x,-\bar{s}, \bar{t}), \\
& \text { (c) } h(x, \bar{s}, \bar{t})=\frac{1}{p^{\prime}} \nabla_{\bar{s}, \bar{t}} h(x, \bar{s}, \bar{t}) \cdot\left(\begin{array}{c}
\bar{s} \\
\bar{t}
\end{array}\right), \\
& \text { (d) } h(x, \bar{s}, 0)=\frac{a(x)^{1-p^{\prime}}}{p^{\prime}}|\bar{s}|^{p^{\prime}} \text { as well as } h(x, \bar{s}, \bar{s})=\frac{2 a(x)^{1-p^{\prime}}}{p^{\prime}}(1+b(x))^{1-p^{\prime}}|\bar{s}|^{p^{\prime}}, \\
& \text { (e) } \frac{1}{p^{\prime}}(a(x)(1+b(x)))^{1-p^{\prime}}\left(|\bar{s}|^{p^{\prime}}+|\bar{t}|^{p^{\prime}}\right) \leq h(x, \bar{s}, \bar{t}) \leq \frac{1}{p^{\prime}} a(x)^{1-p^{\prime}}\left(|\bar{s}|^{p^{\prime}}+|\bar{t}|^{p^{\prime}}\right) .
\end{aligned}
$$

If we additionally impose that the coefficients $a, b: \mathbb{R}^{N} \rightarrow \mathbb{R}$ are measurable, we conclude that for $\bar{u}, \bar{v} \in L^{p^{\prime}}\left(\mathbb{R}^{N}\right)$, the mapping

$$
\begin{aligned}
x \mapsto h(x, \bar{u}(x), \bar{v}(x)) & =\sup _{s, t \in \mathbb{R}}(s \bar{u}(x)+t \bar{v}(x)-f(x, s, t)) \\
& =\sup _{s, t \in \mathbb{Q}}(s \bar{u}(x)+t \bar{v}(x)-f(x, s, t))
\end{aligned}
$$

is measurable since it is a pointwise supremum of countably many measurable functions. Moreover, when combined with (e) of the previous Lemma, we have $h(\cdot, \bar{u}, \bar{v}) \in L^{1}\left(\mathbb{R}^{N}\right)$ and the functional $J_{\mu \nu}$ as introduced in equation (5) is well-defined and continuously Fréchet differentiable. In particular, for $\bar{u}, \bar{v} \in L^{p^{\prime}}\left(\mathbb{R}^{N}\right)$, property (c) yields the identity

$$
J_{\mu \nu}^{\prime}(\bar{u}, \bar{v})[\bar{u}, \bar{v}]=\int_{\mathbb{R}^{N}} p^{\prime} h(x, \bar{u}, \bar{v}) \mathrm{d} x-\int_{\mathbb{R}^{N}} \bar{u} \Psi_{\mu} * \bar{u}+\bar{v} \Psi_{\nu} * \bar{v} \mathrm{~d} x .
$$

\section{Proof of Theorem 1}

In this section, we give the proof of Theorem 1. This will be achieved using the Mountain Pass Theorem and following the ideas in [7]. We endow the product space $L^{p^{\prime}}\left(\mathbb{R}^{N}\right) \times L^{p^{\prime}}\left(\mathbb{R}^{N}\right)$ with the norm given by $\|(\bar{u}, \bar{v})\|_{p^{\prime}}:=\|\bar{u}\|_{p^{\prime}}+\|\bar{v}\|_{p^{\prime}}$ for $\bar{u}, \bar{v} \in L^{p^{\prime}}\left(\mathbb{R}^{N}\right)$ and collect the following two major auxiliary results: 
Lemma 2 (Mountain Pass Geometry, see Lemma 4.2 in [7]). Assuming (7), the functional $J_{\mu \nu}$ has the following properties:

(i) There exist $\delta>0$ and $\rho \in(0,1)$ with the property that, for $(\bar{u}, \bar{v}) \in L^{p^{\prime}}\left(\mathbb{R}^{N}\right) \times L^{p^{\prime}}\left(\mathbb{R}^{N}\right)$, $J_{\mu \nu}(\bar{u}, \bar{v})>0$ if $0<\|(\bar{u}, \bar{v})\|_{p^{\prime}} \leq \rho$ and $J_{\mu \nu}(\bar{u}, \bar{v}) \geq \delta$ if $\|(\bar{u}, \bar{v})\|_{p^{\prime}}=\rho$.

(ii) There exists $\left(\bar{u}_{1}, \bar{v}_{1}\right) \in L^{p^{\prime}}\left(\mathbb{R}^{N}\right) \times L^{p^{\prime}}\left(\mathbb{R}^{N}\right)$ with $\left\|\left(\bar{u}_{1}, \bar{v}_{1}\right)\right\|_{p^{\prime}}>1$ and $J_{\mu \nu}\left(\bar{u}_{1}, \bar{v}_{1}\right)<0$.

(iii) Every Palais-Smale sequence for $J_{\mu \nu}$ is bounded in $L^{p^{\prime}}\left(\mathbb{R}^{N}\right) \times L^{p^{\prime}}\left(\mathbb{R}^{N}\right)$.

Lemma 3 (Existence of Palais-Smale sequence, see Lemma 6.1 in [7]). Assuming (7), there exists a bounded Palais-Smale sequence $\left(\bar{u}_{n}, \bar{v}_{n}\right)_{n \in \mathbb{N}}$ in $L^{p^{\prime}}\left(\mathbb{R}^{N}\right) \times L^{p^{\prime}}\left(\mathbb{R}^{N}\right)$ for $J_{\mu \nu}$ at the level $c_{\mu \nu}$ as given in equation (6).

Up to minor modifications, both results can be proved in the same way as the corresponding scalar results, Lemma 4.2 and Lemma 6.1 in [7] for $N \geq 3$, which also hold for $N=2$ as explained at the beginning of the proof of Theorem 1.3 (b) of [3]. We thus omit the proof.

Proof of Theorem 1. This proof mainly follows the lines of the proof of Theorem 1.3(b) in [3] for $N=2$ and Theorem 6.2 in [7 for $N \geq 3$, respectively, which we will refer to as the scalar case. We will therefore focus on those parts which differ due to the fact that we discuss a system of equations.

Let $\left(\bar{u}_{n}, \bar{v}_{n}\right)_{n \in \mathbb{N}}$ denote a bounded Palais-Smale sequence at the level $c_{\mu \nu}$ which exists by Lemma 3; then w.l.o.g. $\bar{u}_{n} \rightarrow \bar{u}$ and $\bar{v}_{n} \rightarrow \bar{v}$ as $n \rightarrow \infty$ weakly in $L^{p^{\prime}}\left(\mathbb{R}^{N}\right)$. We perform a concentration compactness argument which relies on the periodicity of the coefficients $a, b$.

Step 1: (Nonvanishing.) There exists a ball $B \subseteq \mathbb{R}^{N}$ such that, up to a subsequence and up to translations, $\inf _{n \in \mathbb{N}} \int_{B} h\left(x, \bar{u}_{n}, \bar{v}_{n}\right) \mathrm{d} x>0$.

As in the scalar case, definition (5) and identity (15) imply, as $n \rightarrow \infty$,

$$
\int_{\mathbb{R}^{N}} \bar{u}_{n} \Psi_{\mu} * \bar{u}_{n}+\bar{v}_{n} \Psi_{\nu} * \bar{v}_{n} \mathrm{~d} x=\frac{2 p}{p-2}\left[J_{\mu \nu}\left(\bar{u}_{n}, \bar{v}_{n}\right)-\frac{1}{p^{\prime}} J_{\mu \nu}^{\prime}\left(\bar{u}_{n}, \bar{v}_{n}\right)\left[\bar{u}_{n}, \bar{v}_{n}\right]\right] \rightarrow \frac{2 p}{p-2} \cdot c_{\mu \nu} .
$$

As $c_{\mu \nu}>0$ due to (6) and Lemma 2 (i), we conclude $\lim \sup _{n \rightarrow \infty} \int_{\mathbb{R}^{N}} \bar{u}_{n} \Psi_{\mu} * \bar{u}_{n} \mathrm{~d} x>0$ or $\lim \sup _{n \rightarrow \infty} \int_{\mathbb{R}^{N}} \bar{v}_{n} \Psi_{\nu} * \bar{v}_{n} \mathrm{~d} x>0$. We apply the (scalar) Nonvanishing Theorems, Theorem 3.1 in [3] for $N=2$ and Theorem 3.1 in [7] for $N \geq 3$ to find $R, \zeta>0$ and $\left(x_{n}\right)_{n \in \mathbb{N}} \subseteq \mathbb{R}^{N}$ such that, up to a subsequence, $\int_{B_{R}\left(x_{n}\right)}\left|\bar{u}_{n}\right|^{p^{\prime}}+\left|\bar{v}_{n}\right|^{p^{\prime}} \mathrm{d} x \geq \zeta$ holds for all $n \in \mathbb{N}$. Possibly enlarging the radius $R$, we may w.l.o.g. assume $x_{n} \in \mathbb{Z}^{N}$ for all $n \in \mathbb{N}$. By Lemma 1 (e), and with $\gamma_{p}:=\frac{1}{p^{\prime}}\left(a_{+}\left(1+b_{+}\right)\right)^{1-p^{\prime}}$, we have

$$
\int_{B_{R}\left(x_{n}\right)} h\left(x, \bar{u}_{n}, \bar{v}_{n}\right) \mathrm{d} x \geq \gamma_{p} \zeta \quad \text { for all } n \in \mathbb{N} .
$$

Next, we introduce the shifted functions $\bar{U}_{n}(x):=\bar{u}\left(x_{n}+x\right)$ and $\bar{V}_{n}(x):=\bar{v}\left(x_{n}+x\right)$ for $n \in \mathbb{N}, x \in \mathbb{R}^{N}$. We note that, due to the periodicity of the coefficients $a, b$ and since $x_{n} \in \mathbb{Z}^{N}$, the Legendre transform is invariant under such translations in the sense that

$$
h\left(x, \bar{U}_{n}(x), \bar{V}_{n}(x)\right)=\sup _{s, t \in \mathbb{R}}\left(s \bar{U}_{n}(x)+t \bar{V}_{n}(x)-f(x, s, t)\right)
$$




$$
\begin{aligned}
& =\sup _{s, t \in \mathbb{R}}\left(s \bar{U}_{n}(x)+t \bar{V}_{n}(x)-f\left(x_{n}+x, s, t\right)\right) \\
& =h\left(x_{n}+x, \bar{U}_{n}(x), \bar{V}_{n}(x)\right) \\
& =h\left(x_{n}+x, \bar{u}_{n}\left(x_{n}+x\right), \bar{v}_{n}\left(x_{n}+x\right)\right)
\end{aligned}
$$

for almost all $x \in \mathbb{R}^{N}$ and every $n \in \mathbb{N}$. Thus, and due to (16),

$$
\int_{\mathbb{R}^{N}} h\left(x, \bar{U}_{n}, \bar{V}_{n}\right) \mathrm{d} x=\int_{\mathbb{R}^{N}} h\left(x, \bar{u}_{n}, \bar{v}_{n}\right) \mathrm{d} x \quad \text { and } \int_{B_{R}(0)} h\left(x, \bar{U}_{n}, \bar{V}_{n}\right) \mathrm{d} x \geq \gamma_{p} \zeta .
$$

With that, arguing as in the scalar case, we obtain that $\left(\bar{U}_{n}, \bar{V}_{n}\right)_{n \in \mathbb{N}}$ is a bounded Palais-Smale sequence for $J_{\mu \nu}$ to the level $c_{\mu \nu}$. Hence, w.l.o.g., there exist $\bar{U}, \bar{V} \in L^{p^{\prime}}\left(\mathbb{R}^{N}\right)$ with $\bar{U}_{n} \rightarrow \bar{U}$ and $\bar{V}_{n} \rightarrow \bar{V}$ as $n \rightarrow \infty$ weakly in $L^{p^{\prime}}\left(\mathbb{R}^{N}\right)$.

We intend to prove that

$$
\int_{B_{R}(0)} h\left(x, \bar{U}_{n}, \bar{V}_{n}\right) \mathrm{d} x \rightarrow \int_{B_{R}(0)} h(x, \bar{U}, \bar{V}) \mathrm{d} x \quad \text { as } n \rightarrow \infty
$$

and that hence, due to the inequality in (17)), $(\bar{U}, \bar{V}) \neq(0,0)$. To this end, we need the following auxiliary result:

Step 2: We have $\mathbb{1}_{B_{R}(0)} \cdot \nabla_{\bar{s}, \bar{t}} h\left(\cdot, \bar{U}_{n}, \bar{V}_{n}\right) \rightarrow \mathbb{1}_{B_{R}(0)} \cdot \nabla_{\bar{s}, \bar{t}} h(\cdot, \bar{U}, \bar{V})$ strongly in $L^{p}\left(\mathbb{R}^{N}\right) \times L^{p}\left(\mathbb{R}^{N}\right)$ as $n \rightarrow \infty$.

Let $\varphi, \psi \in L^{p^{\prime}}\left(\mathbb{R}^{N}\right)$ and $\tilde{\varphi}:=\varphi \cdot \mathbb{1}_{B_{R}(0)}, \tilde{\psi}:=\psi \cdot \mathbb{1}_{B_{R}(0)}$. We estimate for $m, n \in \mathbb{N}$

$$
\begin{aligned}
& \left|\int_{\mathbb{R}^{N}}\left(\mathbb{1}_{B_{R}(0)} \cdot \nabla_{\bar{s}, \bar{t}} h\left(\cdot, \bar{U}_{n}, \bar{V}_{n}\right)-\mathbb{1}_{B_{R}(0)} \cdot \nabla_{\bar{s}, \bar{t}} h\left(\cdot, \bar{U}_{m}, \bar{V}_{m}\right)\right) \cdot\left(\begin{array}{c}
\varphi \\
\psi
\end{array}\right) \mathrm{d} x\right| \\
& \quad=\left|J_{\mu \nu}^{\prime}\left(\bar{U}_{n}, \bar{V}_{n}\right)[\tilde{\varphi}, \tilde{\psi}]-J_{\mu \nu}^{\prime}\left(\bar{U}_{m}, \bar{V}_{m}\right)[\tilde{\varphi}, \tilde{\psi}]+\int_{\mathbb{R}^{N}} \tilde{\varphi} \Psi_{\mu} *\left(\bar{U}_{n}-\bar{U}_{m}\right)+\tilde{\psi} \Psi_{\nu} *\left(\bar{V}_{n}-\bar{V}_{m}\right) \mathrm{d} x\right| \\
& \quad \leq C_{n m}\|(\varphi, \psi)\|_{p^{\prime}},
\end{aligned}
$$

where

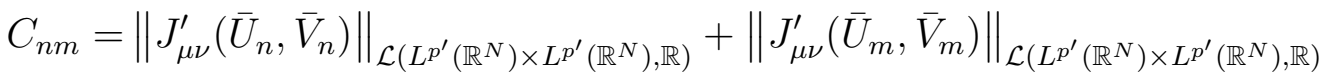

$$
\begin{aligned}
& +\left\|\mathbb{1}_{B_{R}(0)} \cdot \Psi_{\mu} *\left(\bar{U}_{n}-\bar{U}_{m}\right)\right\|_{p}+\left\|\mathbb{1}_{B_{R}(0)} \cdot \Psi_{\nu} *\left(\bar{V}_{n}-\bar{V}_{m}\right)\right\|_{p} .
\end{aligned}
$$

Then, we have $C_{n m} \rightarrow 0$ as $m, n \rightarrow \infty$ since $\left\|J_{\mu \nu}^{\prime}\left(\bar{U}_{n}, \bar{V}_{n}\right)\right\|_{\mathcal{L}\left(L^{p^{\prime}}\left(\mathbb{R}^{N}\right) \times L^{\left.p^{\prime}\left(\mathbb{R}^{N}\right), \mathbb{R}\right)}\right.} \rightarrow 0$ by the Palais-Smale property and since the operator

$$
L^{p^{\prime}}\left(\mathbb{R}^{N}\right) \rightarrow L^{p}\left(\mathbb{R}^{N}\right), \quad g \mapsto \mathbb{1}_{B_{R}(0)} \cdot \Psi_{\mu} * g
$$

is compact, cf. Lemma 4.1 in [7] for $N \geq 3$ and the corresponding result at the beginning of section 3 of [3] for $N=2$.

By duality, $\left(\mathbb{1}_{B_{R}(0)} \cdot \nabla_{\bar{s}, \bar{t}} h\left(\cdot, \bar{U}_{n}, \bar{V}_{n}\right)\right)_{n \in \mathbb{N}}$ is a Cauchy sequence in $L^{p}\left(\mathbb{R}^{N}\right) \times L^{p}\left(\mathbb{R}^{N}\right)$. We thus find $U, V \in L^{p}\left(B_{R}(0)\right)$ with $\nabla_{\bar{s}, \bar{t}} h\left(\cdot, \bar{U}_{n}, \bar{V}_{n}\right) \rightarrow(U, V)$ as $n \rightarrow \infty$ in $L^{p}\left(B_{R}(0)\right) \times L^{p}\left(B_{R}(0)\right)$ and, up to a subsequence, pointwise almost everywhere on $B_{R}(0)$. 
As $\nabla_{s, t} f(x, \cdot, \cdot)$ is a homeomorphism on $\mathbb{R}^{2}$ for all $x \in \mathbb{R}^{N}$, we have $\left(\bar{U}_{n}, \bar{V}_{n}\right) \rightarrow \nabla_{s, t} f(\cdot, U, V)$ almost everywhere on $B_{R}(0)$ as $n \rightarrow \infty$. Since the sequences $\left(\bar{U}_{n}\right)_{n \in \mathbb{N}},\left(\bar{V}_{n}\right)_{n \in \mathbb{N}}$ are bounded in $L^{p^{\prime}}\left(B_{R}(0)\right)$, Theorem 1 in [11] implies that $\left(\bar{U}_{n}, \bar{V}_{n}\right) \rightarrow \nabla_{s, t} f(\cdot, U, V)$ weakly in $L^{p^{\prime}}\left(B_{R}(0)\right) \times$ $L^{p^{\prime}}\left(B_{R}(0)\right)$ as $n \rightarrow \infty$. However, from the end of Step 1 , we know that $\left(\bar{U}_{n}, \bar{V}_{n}\right) \rightarrow(\bar{U}, \bar{V})$ weakly in $L^{p^{\prime}}\left(\mathbb{R}^{N}\right) \times L^{p^{\prime}}\left(\mathbb{R}^{N}\right)$ as $n \rightarrow \infty$. Uniqueness of the weak limit now implies $(\bar{U}, \bar{V})=$ $\nabla_{s, t} f(\cdot, U, V)$ in $L^{p^{\prime}}\left(B_{R}(0)\right) \times L^{p^{\prime}}\left(B_{R}(0)\right)$, hence $(U, V)=\left.\nabla_{\bar{s}, \bar{t}} h(\cdot, \bar{U}, \bar{V})\right|_{B_{R}(0)}$ and

$$
\mathbb{1}_{B_{R}(0)} \cdot \nabla_{\bar{s}, \bar{t}} h\left(\cdot, \bar{U}_{n}, \bar{V}_{n}\right) \rightarrow \mathbb{1}_{B_{R}(0)} \cdot \nabla_{\bar{s}, \bar{t}} h(\cdot, \bar{U}, \bar{V}) \quad \text { as } n \rightarrow \infty \text { in } L^{p}\left(\mathbb{R}^{N}\right) \times L^{p}\left(\mathbb{R}^{N}\right) .
$$

\section{Step 3: Conclusion.}

We find with Lemma 1 (c)

$$
\begin{aligned}
& \left|\int_{B_{R}(0)} h\left(x, \bar{U}_{n}, \bar{V}_{n}\right)-h(x, \bar{U}, \bar{V}) \mathrm{d} x\right| \\
& =\frac{1}{p^{\prime}}\left|\int_{B_{R}(0)} \nabla_{\bar{s}, \bar{t}} h\left(x, \bar{U}_{n}, \bar{V}_{n}\right) \cdot\left(\begin{array}{c}
\bar{U}_{n} \\
\bar{V}_{n}
\end{array}\right)-\nabla_{\bar{s}, \bar{t}} h(x, \bar{U}, \bar{V}) \cdot\left(\begin{array}{c}
\bar{U} \\
\bar{V}
\end{array}\right) \mathrm{d} x\right| \\
& \leq \frac{1}{p^{\prime}}\left\|\mathbb{1}_{B_{R}(0)}\left(\nabla_{\bar{s}, \bar{t}} h\left(\cdot, \bar{U}_{n}, \bar{V}_{n}\right)-\nabla_{\bar{s}, \bar{t}} h(\cdot, \bar{U}, \bar{V})\right)\right\|_{p}\left\|\left(\bar{U}_{n}, \bar{V}_{n}\right)\right\|_{p^{\prime}} \\
& \quad+\frac{1}{p^{\prime}}\left|\int_{B_{R}(0)} \nabla_{\bar{s}, \bar{t}} h(x, \bar{U}, \bar{V}) \cdot\left[\left(\begin{array}{c}
\bar{U}_{n} \\
\bar{V}_{n}
\end{array}\right)-\left(\begin{array}{c}
\bar{U} \\
\bar{V}
\end{array}\right)\right] \mathrm{d} x\right|
\end{aligned}
$$

and both terms tend to zero by Step 2 and Step 1, respectively. Hence, with a view to the inequality in (17), we have

$$
\int_{B_{R}(0)} h(x, \bar{U}, \bar{V}) \mathrm{d} x=\lim _{n \rightarrow \infty} \int_{B_{R}(0)} h\left(x, \bar{U}_{n}, \bar{V}_{n}\right) \mathrm{d} x \geq \gamma_{p} \zeta>0
$$

which shows (via (e) of Lemma 1) that the weak limit satisfies $(\bar{U}, \bar{V}) \neq(0,0)$.

What remains to prove is that indeed $J_{\mu \nu}(\bar{U}, \bar{V})=c_{\mu \nu}$ and $J_{\mu \nu}^{\prime}(\bar{U}, \bar{V})=0$. As in the scalar case, this is a consequence of the fact that $\left(\bar{U}_{n}, \bar{V}_{n}\right)_{n \in \mathbb{N}}$ is a Palais-Smale sequence which converges weakly to $(\bar{U}, \bar{V})$; for details cf. the last lines of the proof of Theorem 6.2 in [7] and of Theorem 1.3 (b) in [3], respectively.

Finally, letting $u:=\partial_{\bar{s}} h(\cdot, \bar{u}, \bar{v})$ and $v:=\partial_{\bar{t}} h(\cdot, \bar{u}, \bar{v})$, it can be shown as in Lemma 4.3 in [7] that this provides a strong solution of (1) and that $u, v \in W^{2, q}\left(\mathbb{R}^{N}\right) \cap C^{1, \alpha}\left(\mathbb{R}^{N}\right)$ for all $p \leq q<\infty, 0<\alpha<1$.

\section{ENERGy LEVELS AND AN INF-SUP CHARACTERIZATION OF A DUAL GROUND STATE}

As announced earlier, the proofs of Theorems 2 and 3 essentially consist of a comparison of energy levels. Thus, the following alternative characterization of the mountain pass level is a crucial ingredient. In preparation, we define $F_{\mu \nu}: L^{p^{\prime}}\left(\mathbb{R}^{N}\right) \times L^{p^{\prime}}\left(\mathbb{R}^{N}\right) \rightarrow(0, \infty]$ by 
$F_{\mu \nu}(0,0):=\infty$ and, for $(\bar{u}, \bar{v}) \in L^{p^{\prime}}\left(\mathbb{R}^{N}\right) \times L^{p^{\prime}}\left(\mathbb{R}^{N}\right) \backslash\{(0,0)\}$,

$$
F_{\mu \nu}(\bar{u}, \bar{v}):=\frac{p-2}{2 p}\left(\frac{\left[\int_{\mathbb{R}^{N}} p^{\prime} h(x, \bar{u}, \bar{v}) \mathrm{d} x\right]^{\frac{1}{p^{\prime}}}}{\left[\int_{\mathbb{R}^{N}} \bar{u} \Psi_{\mu} * \bar{u}+\bar{v} \Psi_{\nu} * \bar{v} \mathrm{~d} x\right]_{+}^{\frac{1}{2}}}\right)^{\frac{2 p}{p-2}} .
$$

With definition (5) and Lemma 1 (a), we have

$$
J_{\mu \nu}(\tau \bar{u}, \tau \bar{v})=\frac{\tau^{p^{\prime}}}{p^{\prime}} \int_{\mathbb{R}^{N}} p^{\prime} h(x, \bar{u}, \bar{v}) \mathrm{d} x-\frac{\tau^{2}}{2} \int_{\mathbb{R}^{N}} \bar{u} \Psi_{\mu} * \bar{u}+\bar{v} \Psi_{\nu} * \bar{v} \mathrm{~d} x
$$

for $\tau>0$. The mapping $\tau \mapsto J_{\mu \nu}(\tau \bar{u}, \tau \bar{v})$ possesses a critical point on $(0, \infty)$ if and only if $\int_{\mathbb{R}^{N}} \bar{u} \Psi_{\mu} * \bar{u}+\bar{v} \Psi_{\nu} * \bar{v} \mathrm{~d} x>0$; in this case, the critical point is unique and a global maximum. A straightforward calculation shows

$$
\sup _{\tau>0} J_{\mu \nu}(\tau \bar{u}, \tau \bar{v})=F_{\mu \nu}(\bar{u}, \bar{v}) .
$$

As a result of one-dimensional calculus, we obtain the following Lemma which provides an inf-sup characterization of the mountain pass level $c_{\mu \nu}$ defined in equation (66). We do not present its proof; variants of it can be found in the literature, e.g. the first lines of the proof of Proposition 2.1 in [14] and the pretext of Lemma 2.1 in [4].

Lemma 4. Under the assumptions given in (7), the mountain pass level $c_{\mu \nu}$ as defined in equation (6) can be characterized as follows:

$$
c_{\mu \nu}=\inf \left\{F_{\mu \nu}(\bar{u}, \bar{v}):(\bar{u}, \bar{v}) \in L^{p^{\prime}}\left(\mathbb{R}^{N}\right) \times L^{p^{\prime}}\left(\mathbb{R}^{N}\right)\right\} .
$$

Moreover, $\left(\bar{u}_{0}, \bar{v}_{0}\right) \in L^{p^{\prime}}\left(\mathbb{R}^{N}\right) \times L^{p^{\prime}}\left(\mathbb{R}^{N}\right)$ is a minimizer of the functional $F_{\mu \nu}$ if and only if it is a nonzero multiple of a critical point of $J_{\mu \nu}$ on the mountain pass level $c_{\mu \nu}$.

These results also apply in the scalar case discussed in Remark 1, we define the functional

$$
E_{\mu}: L^{p^{\prime}}\left(\mathbb{R}^{N}\right) \rightarrow(0, \infty], \quad E_{\mu}(\bar{u}):=\frac{p-2}{2 p}\left(\frac{\left[\int_{\mathbb{R}^{N}} a(x)^{1-p^{\prime}}|\bar{u}|^{p^{\prime}} \mathrm{d} x\right]^{\frac{1}{p^{\prime}}}}{\left[\int_{\mathbb{R}^{N}} \bar{u} \Psi_{\mu} * \bar{u} \mathrm{~d} x\right]_{+}^{\frac{1}{2}}}\right)^{\frac{2 p}{p-2}}
$$

again with $E_{\mu}(0):=+\infty$. Then $E_{\mu}(\bar{u})=F_{\mu \nu}(\bar{u}, 0)$ for $\bar{u} \in L^{p^{\prime}}\left(\mathbb{R}^{N}\right)$ by Lemma 1 (d) and the scalar mountain pass level $c_{\mu}$ is the infimum of $E_{\mu}$, attained in particular at critical points of $I_{\mu}$ on the level $c_{\mu}$. We derive some direct consequences describing the relation between the mountain pass level associated with the system (3) and the scalar mountain pass level. Recall that a critical point of $J_{\mu \nu}$ on the mountain pass level $c_{\mu \nu}$ is said to be a dual ground state.

Lemma 5. We assume that conditions (7) hold. Then we have the following:

(i) The inequality $c_{\mu \nu} \leq c_{\mu}$ holds.

(ii) If $\left(\bar{u}_{0}, 0\right)$ is a semitrivial dual ground state of $J_{\mu \nu}$, then $c_{\mu \nu}=c_{\mu}$ and $\bar{u}_{0}$ is a dual ground state of the scalar functional $I_{\mu}$.

Proof. (i) This is a consequence of the fact that $I_{\mu}(\bar{u})=J_{\mu \nu}(\bar{u}, 0)$ for $\bar{u} \in L^{p^{\prime}}\left(\mathbb{R}^{N}\right)$. 
(ii) Assume that $\left(\bar{u}_{0}, 0\right) \in L^{p^{\prime}}\left(\mathbb{R}^{N}\right) \times L^{p^{\prime}}\left(\mathbb{R}^{N}\right)$ is a dual ground state of $J_{\mu \nu}$, i.e. $J_{\mu \nu}^{\prime}\left(\bar{u}_{0}, 0\right)=$ 0 and $J_{\mu \nu}\left(\bar{u}_{0}, 0\right)=c_{\mu \nu}$. As we have $I_{\mu}(\bar{w})=J_{\mu \nu}(\bar{w}, 0)$ for all $w \in L^{p^{\prime}}\left(\mathbb{R}^{N}\right)$, this implies $I_{\mu}^{\prime}\left(\bar{u}_{0}\right)=0$ and $I_{\mu}\left(\bar{u}_{0}\right)=c_{\mu \nu}$. Then, with (19) and Lemma 4,

$$
c_{\mu}=\inf _{\bar{u} \in L^{p^{\prime}}\left(\mathbb{R}^{N}\right)} E_{\mu}(\bar{u}) \leq E_{\mu}\left(\bar{u}_{0}\right)=F_{\mu \nu}\left(\bar{u}_{0}, 0\right)=c_{\mu \nu} \stackrel{(i)}{\leq} c_{\mu},
$$

we conclude $c_{\mu \nu}=c_{\mu}$ and therefore $\bar{u}_{0}$ is a dual ground state of $I_{\mu}$.

\section{Proof of Theorems 2 and 3}

By $h_{ \pm}: \mathbb{R}^{2} \rightarrow \mathbb{R}$ we denote the Legendre transforms of the functions $f_{ \pm}: \mathbb{R}^{2} \rightarrow \mathbb{R}, f_{ \pm}(s, t):=$ $\frac{1}{p}\left(|s|^{p}+2 b_{ \pm}|s|^{\frac{p}{2}}|t|^{\frac{p}{2}}+|t|^{p}\right)$. We recall that $a_{-} \leq a(x) \leq a_{+}$and $b_{-} \leq b(x) \leq b_{+}$hold for almost all $x \in \mathbb{R}^{N}$. As a direct consequence of Lemma 1 (a), we have the following chain of inequalities for all $\bar{s}, \bar{t} \in \mathbb{R}$ and $x \in \mathbb{R}^{N}$ :

$$
a_{+}^{1-p^{\prime}} h_{+}(\bar{s}, \bar{t}) \leq a(x)^{1-p^{\prime}} h_{+}(\bar{s}, \bar{t}) \leq h(x, \bar{s}, \bar{t}) \leq a(x)^{1-p^{\prime}} h_{-}(\bar{s}, \bar{t}) \leq a_{-}^{1-p^{\prime}} h_{-}(\bar{s}, \bar{t}) .
$$

We recall that, as in Lemma 1 (a), we have for $\bar{s}, \bar{t} \in \mathbb{R}$

$$
h_{ \pm}(\bar{s}, \bar{t})=\frac{1}{p^{\prime}}\left[\sup _{\sigma>0} \frac{|\bar{s}|+\sigma|\bar{t}|}{\left(1+2 b_{ \pm} \sigma^{\frac{p}{2}}+\sigma^{p}\right)^{\frac{1}{p}}}\right]^{p^{\prime}} .
$$

Proof of Theorem Q (a). We consider a minimizer $\bar{w} \in L^{p^{\prime}}\left(\mathbb{R}^{N}\right), \bar{w} \neq 0$ of the scalar functional $E_{\mu}$. Multiplying with a suitable constant, we assume w.l.o.g. $\int_{\mathbb{R}^{N}} \bar{w} \Psi_{\mu} * \bar{w} \mathrm{~d} x=1$ and hence, recalling definition (19),

$$
c_{\mu}=E_{\mu}(\bar{w})=\frac{p-2}{2 p}\left(\int_{\mathbb{R}^{N}} a(x)^{1-p^{\prime}}|\bar{w}|^{p^{\prime}} \mathrm{d} x\right)^{\frac{2(p-1)}{p-2}}=\frac{p-2}{2 p}\left\|a^{-1 / p} \bar{w}\right\|_{p^{\prime}}^{\frac{2 p}{p-2}} .
$$

Theorem 1.1 in [3] for $N=2$ and Lemma 4.3 in [7] for $N \geq 3$, respectively, assure that $\bar{w}$ is continuous. Moreover, equations (6) in [3] and (11), (12) in [7] imply that $\Psi_{\nu}>0$ near zero. Hence there exist $r>0$ and $x_{0} \in \mathbb{R}^{N}$ with $\bar{w}>0$ on $B_{r}\left(x_{0}\right)$ (or with $\bar{w}<0$ on $B_{r}\left(x_{0}\right)$ ) and $\Psi_{\nu}>0$ on $B_{2 r}(0)$. We then have

$$
q:=\int_{\mathbb{R}^{N}}\left(\bar{w} \mathbb{1}_{B_{r}\left(x_{0}\right)}\right) \Psi_{\nu} *\left(\bar{w} \mathbb{1}_{B_{r}\left(x_{0}\right)}\right) \mathrm{d} x=\int_{B_{r}\left(x_{0}\right)} \int_{B_{r}\left(x_{0}\right)} \bar{w}(y) \bar{w}(z) \Psi_{\nu}(y-z) \mathrm{d} y \mathrm{~d} z>0
$$

and estimate for sufficiently small $\eta>0$ :

$$
\begin{aligned}
c_{\mu \nu} & \leq F_{\mu \nu}\left(\bar{w}, \eta \bar{w} \mathbb{1}_{B_{r}\left(x_{0}\right)}\right) \\
& =\frac{p-2}{2 p}\left(\frac{\left(\int_{\mathbb{R}^{N}} p^{\prime} h\left(x, \bar{w}, \eta \bar{w} \mathbb{1}_{B_{r}\left(x_{0}\right)}\right) \mathrm{d} x\right)^{\frac{1}{p^{\prime}}}}{\left(\int_{\mathbb{R}^{N}} \bar{w} \Psi_{\mu} * \bar{w}+\eta^{2}\left(\bar{w} \mathbb{1}_{B_{r}\left(x_{0}\right)}\right) \Psi_{\nu} *\left(\bar{w} \mathbb{1}_{B_{r}\left(x_{0}\right)}\right) \mathrm{d} x\right)_{+}^{\frac{1}{2}}}\right)^{\frac{2 p}{p-2}}
\end{aligned}
$$




$$
\begin{aligned}
& =\frac{p-2}{2 p}\left(\frac{\left(\int_{\mathbb{R}^{N}} p^{\prime} h\left(x, \bar{w}, \eta \bar{w} \mathbb{1}_{B_{r}\left(x_{0}\right)}\right) \mathrm{d} x\right)^{\frac{1}{p^{\prime}}}}{\left(1+\eta^{2} q\right)^{\frac{1}{2}}}\right)^{\frac{2 p}{p-2}} \\
& \stackrel{\text { [20] }}{\leq} \frac{p-2}{2 p}\left(\frac{\left(\int_{\mathbb{R}^{N}} p^{\prime} a(x)^{1-p^{\prime}} h_{-}\left(\bar{w}, \eta \bar{w} \mathbb{1}_{B_{r}\left(x_{0}\right)}\right) \mathrm{d} x\right)^{\frac{1}{p^{\prime}}}}{\left(1+\eta^{2} q\right)^{\frac{1}{2}}}\right)^{\frac{2 p}{p-2}} \\
& \stackrel{\text { (21) }}{=} \frac{p-2}{2 p}\left(\frac{1}{\left(1+\eta^{2} q\right)^{\frac{1}{2}}}\left(\int_{\mathbb{R}^{N}} a(x)^{1-p^{\prime}}\left(\sup _{\sigma>0} \frac{|\bar{w}(x)|+\sigma \eta|\bar{w}(x)| \mathbb{1}_{B_{r}\left(x_{0}\right)}(x)}{\left(1+2 b_{-} \sigma^{\frac{p}{2}}+\sigma^{p}\right)^{\frac{1}{p}}}\right)^{p^{\prime}} \mathrm{d} x\right)^{\frac{1}{p^{\prime}}}\right)^{\frac{2 p}{p-2}} \\
& \leq \frac{p-2}{2 p}\left\|a^{-1 / p} \bar{w}\right\|_{p^{\prime}}^{\frac{2 p}{p-2}} \cdot\left(\sup _{\sigma>0} \frac{1+\sigma \eta}{\left(1+2 b_{-} \sigma^{\frac{p}{2}}+\sigma^{p}\right)^{\frac{1}{p}}\left(1+\eta^{2} q\right)^{\frac{1}{2}}}\right)^{\frac{2 p}{p-2}} \\
& \stackrel{(22)}{=} c_{\mu} \cdot\left(\sup _{\sigma>0} \frac{1+\sigma \eta}{\left(1+2 b_{-} \sigma^{\frac{p}{2}}+\sigma^{p}\right)^{\frac{1}{p}}\left(1+\eta^{2} q\right)^{\frac{1}{2}}}\right)^{\frac{2 p}{p-2}}<c_{\mu} \text {. }
\end{aligned}
$$

The latter estimate holds for sufficiently small positive $\eta$ because we have, with $\tilde{b}_{-}:=$ $\min \left\{1, b_{-}\right\}>0$ and Taylor's Theorem,

$$
\begin{aligned}
\sup _{\sigma>0} \frac{1+\sigma \eta}{\left(1+2 b_{-} \sigma^{\frac{p}{2}}+\sigma^{p}\right)^{\frac{1}{p}}\left(1+\eta^{2} q\right)^{\frac{1}{2}}} & \leq \sup _{\sigma>0} \frac{1+\sigma \eta}{\left(1+\tilde{b}_{-} \sigma^{\frac{p}{2}}\right)^{\frac{2}{p}}\left(1+\eta^{2} q\right)^{\frac{1}{2}}} \\
& =\frac{\left(1+\eta^{\frac{p}{p-2}} \tilde{b}_{-}^{-\frac{2}{p-2}}\right)^{\frac{p-2}{p}}}{\left(1+\eta^{2} q\right)^{\frac{1}{2}}} \\
& =\frac{1+\frac{p-2}{p} \eta^{\frac{p}{p-2}} \tilde{b}_{-}^{-\frac{2}{p-2}}+o\left(\eta^{\frac{p}{p-2}}\right)}{1+\frac{1}{2} \eta^{2} q+o\left(\eta^{2}\right)} \\
& =1-\frac{1}{2} \eta^{2} q+o\left(\eta^{2}\right)
\end{aligned}
$$$$
\text { as } \eta \searrow 0
$$

where we used that $\frac{p}{p-2}>2$ since $2<p<4$. We have shown that $c_{\mu \nu}<c_{\mu}$. Similarly, one proves that $c_{\mu \nu}<c_{\nu}$. Lemma 5 (ii) implies that $J_{\mu \nu}$ cannot have a semitrivial dual ground state.

The proof of Theorem 2 (b) is based on a continuity argument which requires additional knowledge of the scalar case for $a \equiv 1$. Here we let

$$
D_{\lambda}(\bar{w}):=\frac{p-2}{2 p}\left(\frac{\left(\int_{\mathbb{R}^{N}}|\bar{w}|^{p^{\prime}} \mathrm{d} x\right)^{\frac{1}{p^{\prime}}}}{\left(\int_{\mathbb{R}^{N}} \bar{w} \Psi_{\lambda} * \bar{w} \mathrm{~d} x\right)_{+}^{\frac{1}{2}}}\right)^{\frac{2 p}{p-2}}, \quad d_{\lambda}:=\inf _{\bar{w} \in L^{p^{\prime}}\left(\mathbb{R}^{N}\right)} D_{\lambda}(\bar{w})
$$


and, with a view to definition (19), immediately note that

$$
a_{+}^{-\frac{2}{p-2}} d_{\lambda} \leq c_{\lambda} \leq a_{-}^{-\frac{2}{p-2}} d_{\lambda}
$$

Remark 1 guarantees that the functional $I_{1}$ with $a(x) \equiv 1$ admits a dual ground state $\bar{z} \in L^{p^{\prime}}\left(\mathbb{R}^{N}\right)$ which, by the remarks following Lemma 4, is a minimizer of the functional $D_{1}$. We fix such a minimizer $\bar{z}$ and introduce for $\lambda>0$ the rescaled functions

$$
\bar{z}_{\lambda} \in L^{p^{\prime}}\left(\mathbb{R}^{N}\right), \quad \bar{z}_{\lambda}(x):=\lambda^{\frac{N+2}{4}} \bar{z}(\sqrt{\lambda} x), \quad x \in \mathbb{R}^{N} .
$$

Then $\bar{z}_{\lambda}$ is a minimizer of the functional $D_{\lambda}$, and we have

$$
d_{\lambda}=\lambda^{\frac{p}{p-2}-\frac{N}{2}} \cdot d_{1}=\lambda^{\frac{p}{p-2}-\frac{N}{2}} \cdot \frac{p-2}{2 p}\left(\frac{\left[\int_{\mathbb{R}^{N}}|\bar{z}|^{p^{\prime}} \mathrm{d} x\right]^{\frac{1}{p^{\prime}}}}{\left[\int_{\mathbb{R}^{N}} \bar{z} \Psi_{1} * \bar{z} \mathrm{~d} x\right]^{\frac{1}{2}}}\right)^{\frac{2 p}{p-2}} .
$$

The proof of (25) is based on the observation

$$
\Psi_{\lambda}(x)=\lambda^{\frac{N-2}{2}} \Psi_{1}(\sqrt{\lambda} x) \quad \text { and } \quad \int_{\mathbb{R}^{N}} \bar{z}_{\lambda} \Psi_{\lambda} * \bar{z}_{\lambda} \mathrm{d} x=\int_{\mathbb{R}^{N}} \bar{z} \Psi_{1} * \bar{z} \mathrm{~d} x
$$

for $x \in \mathbb{R}^{N}$ and $\lambda>0$, see (12).

Proof of Theorem (2) (b). We aim to prove $c_{\mu \nu}<\min \left\{c_{\mu}, c_{\nu}\right\}$ for sufficiently small values of $\left|\sqrt{\frac{\mu}{\nu}}-1\right|$, which again yields the assertion when applying Lemma 5 (ii). With $\bar{z}, \bar{z}_{\mu}, \bar{z}_{\nu} \in$ $L^{p^{\prime}}\left(\mathbb{R}^{N}\right)$ as above, we estimate as follows:

$$
\begin{aligned}
& c_{\mu \nu} \leq F_{\mu \nu}\left(\bar{z}_{\mu}, \bar{z}_{\nu}\right) \\
& =\frac{p-2}{2 p}\left(\frac{\left[\int_{\mathbb{R}^{N}} p^{\prime} h\left(x, \bar{z}_{\mu}, \bar{z}_{\nu}\right) \mathrm{d} x\right]^{\frac{1}{p^{\prime}}}}{\left[\int_{\mathbb{R}^{N}} \bar{z}_{\mu} \Psi_{\mu} * \bar{z}_{\mu} \mathrm{d} x+\int_{\mathbb{R}^{N}} \bar{z}_{\nu} \Psi_{\nu} * \bar{z}_{\nu} \mathrm{d} x\right]_{+}^{\frac{1}{2}}}\right)^{\frac{2 p}{p-2}} \\
& \stackrel{(26)}{=} \frac{p-2}{2 p}\left(\frac{\left[\int_{\mathbb{R}^{N}} p^{\prime} h\left(x, \bar{z}_{\mu}, \bar{z}_{\nu}\right) \mathrm{d} x\right]^{\frac{1}{p^{\prime}}}}{\left[2 \int_{\mathbb{R}^{N}} \bar{z} \Psi_{1} * \bar{z} \mathrm{~d} x\right]^{\frac{1}{2}}}\right)^{\frac{2 p}{p-2}} \\
& \stackrel{(25)}{=} \frac{d_{1}}{2^{\frac{p}{p-2}}}\left(\frac{\int_{\mathbb{R}^{N}} p^{\prime} h\left(x, \bar{z}_{\mu}, \bar{z}_{\nu}\right) \mathrm{d} x}{\int_{\mathbb{R}^{N}}|\bar{z}|^{p^{\prime}} \mathrm{d} x}\right)^{\frac{2(p-1)}{p-2}} \\
& \stackrel{(20)}{\leq} \frac{d_{1}}{2^{\frac{p}{p-2}}}\left(\frac{a_{-}^{1-p^{\prime}} \int_{\mathbb{R}^{N}} p^{\prime} h_{-}\left(\bar{z}_{\mu}, \bar{z}_{\nu}\right) \mathrm{d} x}{\int_{\mathbb{R}^{N}}|\bar{z}|^{p^{\prime}} \mathrm{d} x}\right)^{\frac{2(p-1)}{p-2}} \\
& \stackrel{\operatorname{Lem} \underset{1}{\leq}}{\leq} \frac{d_{1}}{2^{\frac{p}{p-2}} a_{-}^{\frac{2}{p-2}}}\left(\frac{\int_{\mathbb{R}^{N}} h_{-}\left(\bar{z}_{\mu}, \bar{z}_{\nu}\right) \mathrm{d} x}{\frac{1}{2}\left(1+b_{-}\right)^{\frac{1}{p-1}} \int_{\mathbb{R}^{N}} h_{-}(\bar{z}, \bar{z}) \mathrm{d} x}\right)^{\frac{2(p-1)}{p-2}} \\
& =d_{1} \cdot\left(\frac{2^{\frac{p-2}{2}}}{1+b_{-}} \frac{1}{a_{-}}\right)^{\frac{2}{p-2}}\left(\frac{\int_{\mathbb{R}^{N}} h_{-}\left(\bar{z}_{\mu}, \bar{z}_{\nu}\right) \mathrm{d} x}{\int_{\mathbb{R}^{N}} h_{-}(\bar{z}, \bar{z}) \mathrm{d} x}\right)^{\frac{2(p-1)}{p-2}} .
\end{aligned}
$$


We now introduce $\lambda:=\sqrt{\frac{\mu}{\nu}}$. Then, with $h_{-}(\alpha \bar{s}, \alpha \bar{t})=|\alpha|^{p^{\prime}} h_{-}(\bar{s}, \bar{t})$ (see equation (21)) and substitution:

$$
\begin{aligned}
\left(\int_{\mathbb{R}^{N}} h_{-}\left(\bar{z}_{\mu}, \bar{z}_{\nu}\right) \mathrm{d} x\right)^{\frac{2(p-1)}{p-2}} & =\left(\int_{\mathbb{R}^{N}} h_{-}\left(\mu^{\frac{N+2}{4}} \bar{z}(\sqrt{\mu} x), \nu^{\frac{N+2}{4}} \bar{z}(\sqrt{\nu} x)\right) \mathrm{d} x\right)^{\frac{2(p-1)}{p-2}} \\
& =\left(\int_{\mathbb{R}^{N}} h_{-}\left(\lambda^{\frac{N+2}{2}} \nu^{\frac{N+2}{4}} \bar{z}(\lambda \sqrt{\nu} x), \nu^{\frac{N+2}{4}} \bar{z}(\sqrt{\nu} x)\right) \mathrm{d} x\right)^{\frac{2(p-1)}{p-2}} \\
& =\nu^{\frac{p}{p-2}-\frac{N}{2}}\left(\int_{\mathbb{R}^{N}} h_{-}\left(\lambda^{\frac{N+2}{2}} \bar{z}(\lambda y), \bar{z}(y)\right) \mathrm{d} y\right)^{\frac{2(p-1)}{p-2}} \\
& =\nu^{\frac{p}{p-2}-\frac{N}{2}}\left(\int_{\mathbb{R}^{N}} h_{-}\left(\bar{z}_{\lambda^{2}}, \bar{z}\right) \mathrm{d} y\right)^{\frac{2(p-1)}{p-2}}
\end{aligned}
$$

We insert this into the previous estimate and find

$$
\begin{aligned}
c_{\mu \nu} & \leq d_{1} \nu^{\frac{p}{p-2}-\frac{N}{2}} \cdot\left(\frac{2^{\frac{p-2}{2}}}{1+b_{-}} \frac{1}{a_{-}}\right)^{\frac{2}{p-2}}\left(\frac{\int_{\mathbb{R}^{N}} h_{-}\left(\bar{z}_{\lambda^{2}}, \bar{z}\right) \mathrm{d} x}{\int_{\mathbb{R}^{N}} h_{-}(\bar{z}, \bar{z}) \mathrm{d} x}\right)^{\frac{2(p-1)}{p-2}} \\
& \stackrel{(25)}{=} d_{\nu} \cdot\left(\frac{2^{\frac{p-2}{2}}}{1+b_{-}} \frac{1}{a_{-}}\right)^{\frac{2}{p-2}}\left(\frac{\int_{\mathbb{R}^{N}} h_{-}\left(\bar{z}_{\lambda^{2}}, \bar{z}\right) \mathrm{d} x}{\int_{\mathbb{R}^{N}} h_{-}(\bar{z}, \bar{z}) \mathrm{d} x}\right)^{\frac{2(p-1)}{p-2}} \\
& \stackrel{(23)}{\leq} c_{\nu} \cdot\left(\frac{2^{\frac{p-2}{2}}}{1+b_{-}} \frac{a_{+}}{a_{-}}\right)^{\frac{2}{p-2}}\left(\frac{\int_{\mathbb{R}^{N}} h_{-}\left(\bar{z}_{\lambda^{2}}, \bar{z}\right) \mathrm{d} x}{\int_{\mathbb{R}^{N}} h_{-}(\bar{z}, \bar{z}) \mathrm{d} x}\right)^{\frac{2(p-1)}{p-2}} .
\end{aligned}
$$

Similarly,

$$
c_{\mu \nu} \leq c_{\mu} \cdot\left(\frac{2^{\frac{p-2}{2}}}{1+b_{-}} \frac{a_{+}}{a_{-}}\right)^{\frac{2}{p-2}}\left(\frac{\int_{\mathbb{R}^{N}} h_{-}\left(\bar{z}_{\lambda^{-2}}, \bar{z}\right) \mathrm{d} x}{\int_{\mathbb{R}^{N}} h_{-}(\bar{z}, \bar{z}) \mathrm{d} x}\right)^{\frac{2(p-1)}{p-2}} .
$$

Notice that the terms on the right depend continuously on the parameter $\lambda$ since $\lambda \mapsto$ $\lambda^{\frac{N+2}{2}} \bar{z}(\lambda \cdot)$ is continuous in $L^{p^{\prime}}\left(\mathbb{R}^{N}\right)$. Hence,

$$
\frac{\int_{\mathbb{R}^{N}} h_{-}\left(\bar{z}_{\lambda^{ \pm 2}}, \bar{z}\right) \mathrm{d} x}{\int_{\mathbb{R}^{N}} h_{-}(\bar{z}, \bar{z}) \mathrm{d} x} \rightarrow 1 \quad \text { as } \lambda \rightarrow 1 .
$$

As we have assumed $\frac{2^{\frac{p-2}{2}}}{1+b_{-}} \cdot \frac{a_{+}}{a_{-}}<1$, we find $\delta>0$ such that $|\lambda-1|<\delta$ implies

$$
\left(\frac{2^{\frac{p-2}{2}}}{1+b_{-}} \frac{a_{+}}{a_{-}}\right)^{\frac{2}{p-2}}\left(\frac{\int_{\mathbb{R}^{N}} h_{-}\left(\bar{z}_{\lambda^{ \pm 2}}, \bar{z}\right) \mathrm{d} x}{\int_{\mathbb{R}^{N}} h_{-}(\bar{z}, \bar{z}) \mathrm{d} x}\right)^{\frac{2(p-1)}{p-2}}<1 \quad \text { and hence } \quad c_{\mu \nu}<\min \left\{c_{\mu}, c_{\nu}\right\} .
$$

Lemma 5 (ii) ensures that, for such $\mu$ and $\nu$, every dual ground state is fully nontrivial.

Proof of Theorem 3. We consider a dual ground state $(\bar{u}, \bar{v}) \in L^{p^{\prime}}\left(\mathbb{R}^{N}\right) \times L^{p^{\prime}}\left(\mathbb{R}^{N}\right)$ of the functional $J_{\mu \nu}$, hence a minimizer of $F_{\mu \nu}$, and w.l.o.g. $\bar{u} \neq 0$. We write $\left\|a^{-1 / p} \bar{v}\right\|_{p^{\prime}}=$ 
$\eta_{0}\left\|a^{-1 / p} \bar{u}\right\|_{p^{\prime}}$ for some $\eta_{0} \geq 0$ and aim to show that necessarily $\eta_{0}=0$, hence $\bar{v}=0$.

Recalling that $c_{\mu}, c_{\nu}$ are the minima of $E_{\mu}, E_{\nu}$, respectively, we estimate with (19)

$$
\begin{aligned}
& \left(\int_{\mathbb{R}^{N}} \bar{u} \Psi_{\mu} * \bar{u} \mathrm{~d} x\right)_{+} \leq\left(\frac{2 p}{p-2} c_{\mu}\right)^{-\frac{p-2}{p}}\left\|a^{-1 / p} \bar{u}\right\|_{p^{\prime}}^{2}, \\
& \left(\int_{\mathbb{R}^{N}} \bar{v} \Psi_{\nu} * \bar{v} \mathrm{~d} x\right)_{+} \leq\left(\frac{2 p}{p-2} c_{\nu}\right)^{-\frac{p-2}{p}}\left\|a^{-1 / p} \bar{v}\right\|_{p^{\prime}}^{2} .
\end{aligned}
$$

In the appendix, we will prove the estimate

$$
\int_{\mathbb{R}^{N}} h(x, \bar{u}, \bar{v}) \mathrm{d} x \geq h_{+}\left(\left\|a^{-1 / p} \bar{u}\right\|_{p^{\prime}},\left\|a^{-1 / p} \bar{v}\right\|_{p^{\prime}}\right) .
$$

Both inequalities combined, we have

$$
\begin{aligned}
& c_{\mu \nu}=F_{\mu \nu}(\bar{u}, \bar{v}) \\
& \geq \frac{p-2}{2 p} \frac{\left[p^{\prime} h_{+}\left(\left\|a^{-1 / p} \bar{u}\right\|_{p^{\prime}}, \eta_{0}\left\|a^{-1 / p} \bar{u}\right\|_{p^{\prime}}\right)\right]^{\frac{2(p-1)}{p-2}}}{\left[\left(\frac{2 p}{p-2} c_{\mu}\right)^{-\frac{p-2}{p}}\left\|a^{-1 / p} \bar{u}\right\|_{p^{\prime}}^{2}+\left(\frac{2 p}{p-2} c_{\nu}\right)^{-\frac{p-2}{p}} \eta_{0}^{2}\left\|a^{-1 / p} \bar{u}\right\|_{p^{\prime}}^{2}\right]^{\frac{p}{p-2}}} \\
& {\stackrel{\operatorname{Lem}}{=} \underset{1}{=}\left(p^{\prime}\left\|a^{-1 / p} \bar{u}\right\|_{p^{\prime}}^{p^{\prime}} h_{+}\left(1, \eta_{0}\right)\right]^{\frac{2(p-1)}{p-2}}}_{\left[\left(\left(c_{\mu}\right)^{-\frac{p-2}{p}}+\left(c_{\nu}\right)^{-\frac{p-2}{p}} \eta_{0}^{2}\right)\left\|a^{-1 / p} \bar{u}\right\|_{p^{\prime}}^{2}\right]^{\frac{p}{p-2}}}^{[} \\
& \geq \min \left\{c_{\mu}, c_{\nu}\right\}\left(\frac{\left[p^{\prime} h_{+}\left(1, \eta_{0}\right)\right]^{\frac{1}{p^{\prime}}}}{\left[1+\eta_{0}^{2}\right]^{\frac{1}{2}}}\right)^{\frac{2 p}{p-2}} \\
& \stackrel{(21)}{=} \min \left\{c_{\mu}, c_{\nu}\right\}\left(\sup _{\sigma>0} \frac{\left(1+\sigma \eta_{0}\right)}{\left(1+2 b_{+} \sigma^{\frac{p}{2}}+\sigma^{p}\right)^{\frac{1}{p}}\left(1+\eta_{0}^{2}\right)^{\frac{1}{2}}}\right)^{\frac{2 p}{p-2}} \\
& \geq \min \left\{c_{\mu}, c_{\nu}\right\}\left(\frac{\left(1+\eta_{0}^{2}\right)^{\frac{1}{2}}}{\left(1+2 b_{+} \eta_{0}^{\frac{p}{2}}+\eta_{0}^{p}\right)^{\frac{1}{p}}}\right)^{\frac{2 p}{p-2}} \\
& \geq \min \left\{c_{\mu}, c_{\nu}\right\}\left(\frac{\left(1+\eta_{0}^{2}\right)^{\frac{1}{2}}}{\left(1+\left(2^{\frac{p}{2}}-2\right) \eta_{0}^{\frac{p}{2}}+\eta_{0}^{p}\right)^{\frac{1}{p}}}\right)^{\frac{2 p}{p-2}} \\
& \geq \min \left\{c_{\mu}, c_{\nu}\right\} .
\end{aligned}
$$

The latter estimate holds since

$$
\forall \eta \geq 0 \quad \frac{\left(1+\eta^{2}\right)^{\frac{1}{2}}}{\left(1+\left(2^{\frac{p}{2}}-2\right) \eta^{\frac{p}{2}}+\eta^{p}\right)^{\frac{1}{p}}} \geq 1,
$$


which we will prove in the appendix. Lemma 5 (ii) yields $c_{\mu \nu} \leq \min \left\{c_{\mu}, c_{\nu}\right\}$, and thus we have $c_{\mu \nu}=\min \left\{c_{\mu}, c_{\nu}\right\}$ and equality must hold in all above estimates. But then, since we assume $b_{+}<2^{\frac{p-2}{2}}-1$, we infer $\eta_{0}=0$. Hence, the dual ground state is semitrivial with $\bar{v}=0$.

Proof of Corollary 1. The previously proved Theorems cover most cases: If $2<p<$ 4 and $b>0$, Theorem 2 (a) states that every dual ground state of $J_{\mu \mu}$ is fully nontrivial; so does Theorem 2 (b) in case $p \geq 4$ and $b>2^{\frac{p-2}{2}}-1$. (Notice that we assume $\nu=\mu$.) If, however, $p \geq 4$ and $0 \leq b<2^{\frac{p-2}{2}}-1$, Theorem 3 assures that dual ground states of $J_{\mu \mu}$ are semitrivial. So only two cases remain open.

Assume $2<p<4$ and $b=0$.

The proof then follows the lines of that of Theorem 3. Considering a dual ground state $(\bar{u}, \bar{v}) \in L^{p^{\prime}}\left(\mathbb{R}^{N}\right) \times L^{p^{\prime}}\left(\mathbb{R}^{N}\right)$ of $J_{\mu \nu}$ and assuming $\left\|a^{-1 / p} \bar{v}\right\|_{p^{\prime}}=\eta_{0}\left\|a^{-1 / p} \bar{u}\right\|_{p^{\prime}}$, we again aim to prove that $\eta_{0}=0$. The same estimate as in the previous proof yields

$$
c_{\mu \nu} \geq \min \left\{c_{\mu}, c_{\nu}\right\} \sup _{\sigma>0} \frac{\left(1+\sigma \eta_{0}\right)}{\left(1+\sigma^{p}\right)^{\frac{1}{p}}\left(1+\eta_{0}^{2}\right)^{\frac{1}{2}}} \geq \min \left\{c_{\mu}, c_{\nu}\right\} \frac{\left(1+\eta_{0}^{p^{\prime}}\right)^{\frac{1}{p^{\prime}}}}{\left(1+\eta_{0}^{2}\right)^{\frac{1}{2}}} \stackrel{\left(p^{\prime}<2\right)}{\geq} \min \left\{c_{\mu}, c_{\nu}\right\}
$$

with equality if and only if $\eta_{0}=0$. Since $c_{\mu \nu} \leq \min \left\{c_{\mu}, c_{\nu}\right\}$ by Lemma 5 (i), this implies $\eta_{0}=0$.

Assume $p \geq 4$ and $b=2^{\frac{p-2}{2}}-1$.

In this case, one can show as in the proof of Theorem 3 that we have $c_{\mu \mu}=c_{\mu}$. For any scalar dual ground state $\bar{w} \in L^{p^{\prime}}\left(\mathbb{R}^{N}\right)$ of $I_{\mu}$, we calculate

$$
\begin{aligned}
F_{\mu \mu}(\bar{w}, \bar{w}) & \stackrel{[18]}{=} \frac{p-2}{2 p}\left(\frac{\left[\int_{\mathbb{R}^{N}} p^{\prime} h(x, \bar{w}, \bar{w}) \mathrm{d} x\right]^{\frac{1}{p^{\prime}}}}{\left[\int_{\mathbb{R}^{N}} \bar{w} \Psi_{\mu} * \bar{w}+\bar{w} \Psi_{\mu} * \bar{w} \mathrm{~d} x\right]_{+}^{\frac{1}{2}}}\right)^{\frac{2 p}{p-2}} \\
& \stackrel{\text { Lem. } 11}{=} d) \frac{p-2}{2 p}\left(\frac{\left[\int_{\mathbb{R}^{N}} 2(1+b)^{1-p^{\prime}} a(x)^{1-p^{\prime}}|\bar{w}(x)|^{p^{\prime}} \mathrm{d} x\right]^{\frac{1}{p^{\prime}}}}{\left[2 \int_{\mathbb{R}^{N}} \bar{w} \Psi_{\mu} * \bar{w}\right]_{+}^{\frac{1}{2}}}\right)^{\frac{2 p}{p-2}} \\
& =\frac{2}{(1+b)^{\frac{2}{p-2}}} \frac{p-2}{2 p}\left(\frac{\left[\int_{\mathbb{R}^{N}} a(x)^{1-p^{\prime}}|\bar{w}(x)|^{p^{\prime}} \mathrm{d} x\right]^{\frac{1}{p^{\prime}}}}{\left[\int_{\mathbb{R}^{N}} \bar{w} \Psi_{\mu} * \bar{w}\right]_{+}^{\frac{1}{2}}}\right)^{\frac{2 p}{p-2}} \\
& =\frac{2}{(1+b)^{\frac{2}{p-2}}} E_{\mu}(\bar{w}) \\
& =E_{\mu}(\bar{w}) \\
& =c_{\mu}
\end{aligned}
$$


and $F_{\mu \mu}(\bar{w}, 0)=E_{\mu}(\bar{w})=c_{\mu}$. Hence, $F_{\mu \mu}(\bar{w}, \bar{w})=F_{\mu \mu}(\bar{w}, 0)=c_{\mu \mu}$ and by Lemma 4, this provides (up to multiplication with suitable constants) both a semitrivial and a fully nontrivial dual ground state of $J_{\mu \mu}$.

\section{Appendix}

6.1. Proof of Proposition 1. Fix $x \in \mathbb{R}^{N}$ and recall for $s, t \in \mathbb{R}$

$$
f(x, s, t)=\frac{a(x)}{p}\left(|s|^{p}+2 b(x)|s|^{\frac{p}{2}}|t|^{\frac{p}{2}}+|t|^{p}\right) .
$$

Differentiability and co-finiteness of $f(x, \cdot, \cdot)$ are a straightforward consequence of the assumption $p>2$. We will show below that $f(x, \cdot, \cdot)$ is strictly convex; with that, the existence and the asserted properties of the Legendre transform $h(x, \cdot, \cdot)$ of $f(x, \cdot, \cdot)$ are guaranteed by Theorem 4. To verify strict convexity, we show that, for all $s_{1}, s_{2}, t_{1}, t_{2} \in \mathbb{R}$ with $s_{2} \neq 0$ or $t_{2} \neq 0$,

$$
f\left(x, s_{1}+s_{2}, t_{1}+t_{2}\right)>f\left(x, s_{1}, t_{1}\right)+s_{2} \partial_{s} f\left(x, s_{1}, t_{1}\right)+t_{2} \partial_{t} f\left(x, s_{1}, t_{1}\right) .
$$

We denote the difference by

$$
\mathcal{I}:=f\left(x, s_{1}+s_{2}, t_{1}+t_{2}\right)-\left[f\left(x, s_{1}, t_{1}\right)+s_{2} \partial_{s} f\left(x, s_{1}, t_{1}\right)+t_{2} \partial_{t} f\left(x, s_{1}, t_{1}\right)\right] .
$$

So if we prove $\mathcal{I}>0$, we conclude (29). We introduce the line segment

$$
\ell:=\left\{\left(s_{1}, t_{1}\right)+\theta\left(s_{2}, t_{2}\right) \in \mathbb{R}^{2}: 0 \leq \theta \leq 1\right\} .
$$

(I) Let us assume that $\ell$ is a subset of either of the sets

$$
\begin{array}{ll}
\left\{(s, 0) \in \mathbb{R}^{2}: s \in \mathbb{R}\right\}, & \left\{(0, t) \in \mathbb{R}^{2}: t \in \mathbb{R}\right\}, \\
\left\{(s, s) \in \mathbb{R}^{2}: s \in \mathbb{R}\right\}, & \left\{(s,-s) \in \mathbb{R}^{2}: s \in \mathbb{R}\right\} .
\end{array}
$$

We then conclude $\mathcal{I}>0$ since the functions

$$
\begin{array}{ll}
s \mapsto f(x, s, 0)=\frac{a(x)}{p}|s|^{p}, & t \mapsto f(x, 0, t)=\frac{a(x)}{p}|t|^{p}, \\
s \mapsto f(x, s, s)=\frac{2 a(x)(1+b(x))}{p}|s|^{p}, & s \mapsto f(x, s,-s)=\frac{2 a(x)(1+b(x))}{p}|s|^{p},
\end{array}
$$

respectively, are strictly convex.

(II) We now assume that $\ell$ intersects none of the sets in (30). Then $f$ is twice continuously differentiable in a neighborhood of $\ell$, and the Fundamental Theorem of Calculus yields the integral representation

$$
\mathcal{I}=\int_{0}^{1} \int_{0}^{1} \tau\left(s_{2}, t_{2}\right) D_{s, t}^{2} f\left(x, s_{1}+\tau \sigma s_{2}, t_{1}+\tau \sigma t_{2}\right)\left(\begin{array}{c}
s_{2} \\
t_{2}
\end{array}\right) \mathrm{d} \sigma \mathrm{d} \tau .
$$

We show that the Hessian $D_{s, t}^{2} f(x, s, t)$ is strictly positive definite for all $(s, t) \in \ell$.

Let $(s, t) \in \ell$, i.e. in particular $s \neq 0, t \neq 0$ and $|s| \neq|t|$. Recall that we assume $a(x)>0$ and $0 \leq b(x) \leq p-1$. We calculate the trace and the determinant of the 
Hessian:

$\operatorname{tr} D_{s, t}^{2} f(x, s, t)$

$$
=a(x)(p-1)\left(|s|^{p-2}+|t|^{p-2}\right)+a(x) \frac{b(x)}{2}(p-2)\left(|s|^{\frac{p}{2}-2}|t|^{\frac{p}{2}}+|t|^{\frac{p}{2}-2}|s|^{\frac{p}{2}}\right),
$$

$\operatorname{det} D_{s, t}^{2} f(x, s, t)$

$$
=a(x)^{2}(p-1)\left[\left(p-1-b(x)^{2}\right)|s|^{\frac{p}{2}}|t|^{\frac{p}{2}}+\frac{b(x)}{2}(p-2)\left(|s|^{p}+|t|^{p}\right)\right]|s|^{\frac{p}{2}-2}|t|^{\frac{p}{2}-2} .
$$

Since $a(x)>0 b(x) \geq 0, s \neq 0$ and $t \neq 0$, we always have $\operatorname{tr} D_{s, t}^{2} f(x, s, t)>0$. If $0 \leq b(x) \leq \sqrt{p-1}$, we infer $\operatorname{det} D_{s, t}^{2} f(x, s, t)>0$ and hence $D_{s, t}^{2} f(s, t)$ is strictly positive semidefinite. Else if $\sqrt{p-1}<b(x) \leq p-1$, we recall that $|s| \neq|t|$ by assumption on $\ell$, which gives the strict estimate $|s|^{\frac{p}{2}}|t|^{\frac{p}{2}}<\frac{1}{2}\left(|s|^{p}+|t|^{p}\right)$. Thus,

$$
\begin{aligned}
\operatorname{det} D_{s, t}^{2} f(x, s, t) & >a(x)^{2} \frac{p-1}{2}\left(\left(p-1-b(x)^{2}\right)+b(x)(p-2)\right)\left(|s|^{p}+|t|^{p}\right)|s|^{\frac{p}{2}-2}|t|^{\frac{p}{2}-2} \\
& =a(x)^{2} \frac{(p-1)(b(x)+1)}{2}(p-1-b(x))\left(|s|^{p}+|t|^{p}\right)|s|^{\frac{p}{2}-2}|t|^{\frac{p}{2}-2} \\
& \geq 0
\end{aligned}
$$

which proves strict positive definiteness of $D_{s, t}^{2} f(x, s, t)$.

(III) Finally, in all remaining cases, $\ell$ intersects the sets of (30) in at most finitely many points. Then still, the integral in (31) converges, the integral representation from (31) holds and the previous step gives $\mathcal{I}>0$.

Hence, $f(x, \cdot, \cdot)$ is strictly convex, which concludes the proof.

6.2. Proof of Lemma 1, For $\bar{s}, \bar{t} \in \mathbb{R}$, we recall the definition of the Legendre transform:

$$
h(x, \bar{s}, \bar{t})=\sup _{s, t \in \mathbb{R}}(s \bar{s}+t \bar{t}-f(x, s, t))
$$

where $f(x, s, t)=\frac{a(x)}{p}\left(|s|^{p}+2 b(x)|s|^{\frac{p}{2}}|t|^{\frac{p}{2}}+|t|^{p}\right)$. We note that, since $f(x, s, t) \geq 0$, this immediately yields $h(x, 0,0)=0$.

(a) We assume w.l.o.g. that $\bar{s} \neq 0$. With that,

$$
\begin{aligned}
h(x, \bar{s}, \bar{t}) & =\sup _{s, t \in \mathbb{R}}[s \bar{s}+t \bar{t}-f(x, s, t)] \stackrel{f(x, s, t) \geq 0}{=} \sup _{s, t>0}[s|\bar{s}|+t|\bar{t}|-f(x, s, t)] \\
& =\sup _{s, \sigma>0}\left[s(|\bar{s}|+\sigma|\bar{t}|)-s^{p} f(x, 1, \sigma)\right]=\sup _{\sigma>0} \frac{1}{p^{\prime}}\left(\frac{(|\bar{s}|+\sigma|\bar{t}|)^{p}}{p f(x, 1, \sigma)}\right)^{\frac{1}{p-1}} \\
& =\frac{a(x)^{1-p^{\prime}}}{p^{\prime}}\left[\sup _{\sigma>0} \frac{|\bar{s}|+\sigma|\bar{t}|}{\left(1+2 b(x) \sigma^{\frac{p}{2}}+\sigma^{p}\right)^{\frac{1}{p}}}\right]^{p^{\prime}}
\end{aligned}
$$

where the supremum with respect to $s>0$ has been evaluated explicitly. 
(b) This is a direct consequence of the symmetry of $f(x, \cdot, \cdot)$, i.e. $f(x, s, t)=f(x, t, s)$ and of the fact that $f(x,-s, t)=f(x, s, t)$, respectively, for all $s, t \in \mathbb{R}$.

(c) As a consequence of part (a), we have $h(x, \alpha \bar{s}, \alpha \bar{t})=\alpha^{p^{\prime}} h(x, \bar{s}, \bar{t})$ for $\alpha>0$. We differentiate with respect to $\alpha$ and find

$$
\nabla_{\bar{s}, \bar{t}} h(x, \alpha \bar{s}, \alpha \bar{t}) \cdot\left(\begin{array}{c}
\bar{s} \\
\bar{t}
\end{array}\right)=p^{\prime} \alpha^{p^{\prime}-1} h(x, \bar{s}, \bar{t}) .
$$

Evaluating the latter identity at $\alpha=1$, the assertion of (c) is proved.

(d) We only prove the second identity, the first one can be shown in the same way. By direct computation we find $\nabla_{s, t} f(x, s, s)=a(x)(1+b(x))|s|^{p-2} s(1,1)$ for $s \in \mathbb{R}$. Recalling that $\nabla_{\bar{s}, \bar{t}} h(x, \cdot, \cdot)$ is a diffeomorphism on $\mathbb{R}^{2}$ with inverse $\nabla_{s, t} f(x, \cdot, \cdot)$, this implies $\nabla_{\bar{s}, \bar{t}} h(x, \bar{s}, \bar{s})=(a(x)(1+b(x)))^{-\left(p^{\prime}-1\right)}|\bar{s}|^{p^{\prime}-2} \bar{s}(1,1)$, and hence using (c)

$$
h(x, \bar{s}, \bar{s})=\frac{1}{p^{\prime}} \nabla_{\bar{s}, \bar{t}} h(x, \bar{s}, \bar{s}) \cdot\left(\begin{array}{c}
\bar{s} \\
\bar{s}
\end{array}\right)=\frac{2}{p^{\prime}}(a(x)(1+b(x)))^{1-p^{\prime}}|\bar{s}|^{p^{\prime}} .
$$

(e) We have by definition of the Legendre transform and due to $a(x), b(x) \geq 0$

$$
\begin{aligned}
h(x, \bar{s}, \bar{t}) & =\sup _{s, t \in \mathbb{R}}\left(s \bar{s}+t \bar{t}-\frac{a(x)}{p}\left(|s|^{p}+2 b(x)|s|^{\frac{p}{2}}|t|^{\frac{p}{2}}+|t|^{p}\right)\right) \\
& \leq \sup _{s, t \in \mathbb{R}}\left(s \bar{s}+t \bar{t}-\frac{a(x)}{p}\left(|s|^{p}+|t|^{p}\right)\right)=\frac{1}{p^{\prime}} a(x)^{1-p^{\prime}}\left(|\bar{s}|^{p^{\prime}}+|\bar{t}|^{p^{\prime}}\right)
\end{aligned}
$$

where we calculated the latter supremum explicitly. On the other hand, defining $s_{x} \in \mathbb{R}$ via

$$
s_{x}:=(a(x)(1+b(x)))^{1-p^{\prime}} \cdot|\bar{s}|^{p^{\prime}-2} \bar{s}
$$

we notice that $s_{x}$ maximizes the map $\mathbb{R} \rightarrow \mathbb{R}, s \mapsto s \bar{s}-\frac{1}{p} a(x)(1+b(x))|s|^{p}$ and that $\bar{s}=a(x)(1+b(x))\left|s_{x}\right|^{p-2} s_{x}$. Defining $t_{x} \in \mathbb{R}$ similarly, we estimate

$$
\begin{aligned}
& \frac{1}{p^{\prime}}(a(x)(1+b(x)))^{1-p^{\prime}}\left(|\bar{s}|^{p^{\prime}}+|\bar{t}|^{p^{\prime}}\right) \\
& \quad=\left(1-\frac{1}{p}\right)\left(s_{x} \bar{s}+t_{x} \bar{t}\right) \\
& \quad \leq s_{x} \bar{s}+t_{x} \bar{t}-\frac{1}{p}\left(s_{x} \cdot a(x)(1+b(x))\left|s_{x}\right|^{p-2} s_{x}+t_{x} \cdot a(x)(1+b(x))\left|t_{x}\right|^{p-2} t_{x}\right) \\
& \quad=s_{x} \bar{s}+t_{x} \bar{t}-\frac{a(x)}{p}\left(\left|s_{x}\right|^{p}+2 b(x)\left|s_{x}\right|^{\frac{p}{2}}\left|t_{x}\right|^{\frac{p}{2}}+\left|t_{x}\right|^{p}\right) \\
& \quad \leq \sup _{s, t \in \mathbb{R}}\left(s \bar{s}+t \bar{t}-\frac{a(x)}{p}\left(|s|^{p}+2 b(x)|s|^{\frac{p}{2}}|t|^{\frac{p}{2}}+|t|^{p}\right)\right) \\
& \quad=h(x, \bar{s}, \bar{t}) .
\end{aligned}
$$


6.3. Proof of equation (27). Recall that $h_{+}$was defined to be the Legendre transform of $f_{+}$and $f_{+}(s, t)=\frac{1}{p}\left(|s|^{p}+2 b_{+}|s|^{\frac{p}{2}}|t|^{\frac{p}{2}}+|t|^{p}\right)$. For $\bar{u}, \bar{v} \in L^{p^{\prime}}\left(\mathbb{R}^{N}\right)$, we prove the inequality

$$
\int_{\mathbb{R}^{N}} h(x, \bar{u}, \bar{v}) \mathrm{d} x \geq h_{+}\left(\left\|a^{-1 / p} \bar{u}\right\|_{p^{\prime}},\left\|a^{-1 / p} \bar{v}\right\|_{p^{\prime}}\right) .
$$

By definition of the Legendre transform, we have for $x \in \mathbb{R}^{N}$

$$
h(x, \bar{u}(x), \bar{v}(x))=\sup _{s, t \in \mathbb{R}}[s \bar{u}(x)+t \bar{v}(x)-f(x, s, t)] .
$$

In order to estimate the supremum, we insert explicitly

$$
\begin{aligned}
s_{x} & :=\frac{\sigma}{\left\|a^{-1 / p} \bar{u}\right\|_{p^{\prime}}^{p^{\prime}-1}} \cdot a(x)^{1-p^{\prime}}|\bar{u}(x)|^{p^{\prime}-2} \bar{u}(x), \\
t_{x} & :=\frac{\tau}{\left\|a^{-1 / p} \bar{v}\right\|_{p^{\prime}}^{p^{\prime}-1}} \cdot a(x)^{1-p^{\prime}}|\bar{v}(x)|^{p^{\prime}-2} \bar{v}(x)
\end{aligned}
$$

where $\sigma, \tau \in \mathbb{R}$ are arbitrary. With that, we integrate, estimate $b(x) \leq b_{+}$and apply Hölder's inequality:

$$
\begin{aligned}
& \int_{\mathbb{R}^{N}} h(x, \bar{u}(x), \bar{v}(x)) \mathrm{d} x \\
& \geq \int_{\mathbb{R}^{N}} s_{x} \bar{u}(x)+t_{x} \bar{v}(x)-\frac{a(x)}{p}\left(\left|s_{x}\right|^{p}+2 b(x)\left|s_{x}\right|^{\frac{p}{2}}\left|t_{x}\right|^{\frac{p}{2}}+\left|t_{x}\right|^{p}\right) \mathrm{d} x \\
& =\sigma\left\|a^{-1 / p} \bar{u}\right\|_{p^{\prime}}+\tau\left\|a^{-1 / p} \bar{v}\right\|_{p^{\prime}} \\
& -\frac{1}{p}\left(|\sigma|^{p}+2 b_{+} \frac{|\sigma \tau|^{\frac{p}{2}}}{\left\|a^{-1 / p} \bar{u}\right\|_{p^{\prime}}^{\frac{p^{\prime}}{2}}\left\|a^{-1 / p} \bar{v}\right\|_{p^{\prime}}^{\frac{p^{\prime}}{2}}} \cdot \int_{\mathbb{R}^{N}}\left(a(x)^{-\frac{1}{p}}|\bar{u}|\right)^{\frac{p^{\prime}}{2}}\left(a(x)^{-\frac{1}{p}}|\bar{v}|\right)^{\frac{p^{\prime}}{2}} \mathrm{~d} x+|\tau|^{p}\right) \\
& \geq \sigma\left\|a^{-1 / p} \bar{u}\right\|_{p^{\prime}}+\tau\left\|a^{-1 / p} \bar{v}\right\|_{p^{\prime}}-\frac{1}{p}\left(|\sigma|^{p}+2 b_{+}|\sigma \tau|^{\frac{p}{2}}+|\tau|^{p}\right) \\
& =\sigma\left\|a^{-1 / p} \bar{u}\right\|_{p^{\prime}}+\tau\left\|a^{-1 / p} \bar{v}\right\|_{p^{\prime}}-f_{+}(\sigma, \tau) .
\end{aligned}
$$

Passing to the supremum with respect to $\sigma, \tau \in \mathbb{R}$, we find the asserted inequality.

6.4. Proof of equation (28). Let $p>4$ and consider, for $\eta \geq 0$,

$$
\psi(\eta):=\frac{\left(1+\eta^{2}\right)^{\frac{1}{2}}}{\left(1+\left(2^{\frac{p}{2}}-2\right) \eta^{\frac{p}{2}}+\eta^{p}\right)^{\frac{1}{p}}} .
$$

We assert that $\psi$ has exactly three critical points on $(0, \infty)$ which are given by $\left\{\eta_{1}, 1, \eta_{1}^{-1}\right\}$ for some $\eta_{1} \in(0,1)$, and that $\psi$ attains its minimum on $(0, \infty)$ uniquely at $\eta_{0}=1$.

We note that $\psi$ is smooth on $(0, \infty)$, and that $\psi(\eta) \rightarrow 1$ as $\eta \searrow 0$ or $\eta \nearrow \infty$. Moreover, $\psi\left(\eta^{-1}\right)=\psi(\eta)$ holds for all $\eta>0$. Critical points of $\psi$ satisfy

$$
0=\psi^{\prime}(\eta) \text {, equivalently } 1+\left(2^{\frac{p-2}{2}}-1\right) \eta^{\frac{p}{2}}=\eta^{p-2}+\left(2^{\frac{p-2}{2}}-1\right) \eta^{\frac{p-4}{2}} .
$$


Obviously, this is satisfied for $\eta_{0}:=1$. Moreover, $p>4$ implies that $\psi^{\prime \prime}(1)=\frac{2^{\frac{p}{2}}-p}{2 \cdot 2^{\frac{p}{2}}}>0$, which proves that $\psi(1)=1$ is a strict local minimum. Once we have established that $\psi$ has a unique critical point $\eta_{1}$ in the interval $(0,1)$, we colclude that $\psi$ attains local maxima at $\eta_{1}$ and at $\eta_{1}^{-1}$ and hence that the local minimum in $\eta_{0}=1$ is in fact global.

We substitute $\kappa:=2^{\frac{p-2}{2}}-1(>1), \sigma:=\frac{p-4}{p} \in(0,1), y:=\eta^{\frac{p}{2}}$ and (32a) gives

$$
0=\psi^{\prime}\left(y^{\frac{2}{p}}\right) \Leftrightarrow \frac{1+\kappa y}{\kappa+y}=y^{\sigma} .
$$

Existence of $\eta_{1}$ : This is guaranteed by the Mean Value Theorem since $\psi(0)=\psi(1)=1$.

Uniqueness of $\eta_{1}$ : Now assume that $\psi$ possesses (at least) two critical points $\eta_{1}, \eta_{2}$ in $(0,1)$ with $0<\eta_{1}<\eta_{2}<1$; then $\frac{1}{\eta_{2}}, \frac{1}{\eta_{1}} \in(1, \infty)$ are two more critical points. We denote $y_{j}:=\eta_{j}^{\frac{p}{2}}$ for $j=0,1,2$. Notice that, by (32b) , we have

$$
\frac{1+\kappa y}{\kappa+y}-y^{\sigma}=0 \quad \text { for } y \in\left\{y_{1}, y_{2}, 1, \frac{1}{y_{1}}, \frac{1}{y_{2}}\right\} \text {. }
$$

The Mean Value Theorem yields $z_{1} \in\left(y_{1}, y_{2}\right), z_{2} \in\left(y_{2}, 1\right), z_{3} \in\left(1, \frac{1}{y_{2}}\right), z_{4} \in\left(\frac{1}{y_{2}}, \frac{1}{y_{1}}\right)$ with

$$
\left.\frac{\mathrm{d}}{\mathrm{d} y}\right|_{y=z_{j}}\left(\frac{1+\kappa y}{\kappa+y}-y^{\sigma}\right)=0, \quad \text { equivalently } \sqrt{\frac{\sigma}{\kappa^{2}-1}}\left(\kappa+z_{j}\right)=z_{j}^{\frac{1-\sigma}{2}} .
$$

Then again, we find $z_{1}^{*} \in\left(z_{1}, z_{2}\right)$ and $z_{2}^{*} \in\left(z_{3}, z_{4}\right)$ satisfying

$$
\left.\frac{\mathrm{d}}{\mathrm{d} y}\right|_{y=z_{j}^{*}}\left(\sqrt{\frac{\sigma}{\kappa^{2}-1}}(\kappa+y)-y^{\frac{1-\sigma}{2}}\right)=0, \quad \text { equivalently } \quad \frac{(1-\sigma)^{2}\left(\kappa^{2}-1\right)}{4 \sigma}=\left(z_{j}^{*}\right)^{\sigma+1} .
$$

The latter equation, however, possesses a unique positive solution; since we have found two distinct ones $z_{1}^{*} \in(0,1), z_{2}^{*} \in(1, \infty)$, we have a contradiction.

\section{ACKNOWLEDGEMENTS}

The authors thank Gilles Evéquoz (University of Frankfurt) for stimulating discussions and explanations about the subject as well as for the improvements explained in Remark 3. Both authors gratefully acknowledge financial support by the Deutsche Forschungsgemeinschaft (DFG) through CRC 1173 "Wave phenomena: analysis and numerics".

\section{REFERENCES}

[1] A. Ambrosetti and E. Colorado. Bound and ground states of coupled nonlinear Schrödinger equations. Comptes Rendus Mathematique, 342(7):453 - 458, 2006.

[2] G. Evéquoz. A dual approach in Orlicz spaces for the nonlinear Helmholtz equation. Zeitschrift für angewandte Mathematik und Physik, 66(6):2995 - 3015, 2015.

[3] G. Evéquoz. Existence and asymptotic behavior of standing waves of the nonlinear Helmholtz equation in the plane. arXiv:1606.00788v2, 2016.

[4] G. Evéquoz. Multiple standing waves for the nonlinear Helmholtz equation concentrating in the high frequency limit. arXiv:1608.04534v1, 2016. 
[5] G. Evéquoz. On the periodic and asymptotically periodic nonlinear Helmholtz equation. Nonlinear Analysis: Theory, Methods \& Applications, 152(Supplement C):88 - 101, 2017.

[6] G. Evéquoz and T. Weth. Real Solutions to the Nonlinear Helmholtz Equation with Local Nonlinearity. Archive for Rational Mechanics and Analysis, 211(2):359 - 388, 2014.

[7] G. Evéquoz and T. Weth. Dual variational methods and nonvanishing for the nonlinear Helmholtz equation. Advances in Mathematics, 280:690 - 728, 2015.

[8] G. Evéquoz and T. Weth. Branch continuation inside the essential spectrum for the nonlinear Schrödinger equation. Journal of Fixed Point Theory and Applications, 19(1):475 - 502, 2017.

[9] G. Evéquoz and T. Yeşil. Dual ground state solutions for the critical nonlinear Helmholtz equation. arXiv:1707.00959v1, 2017.

[10] S. Gutiérrez. Non trivial $l^{q}$ solutions to the Ginzburg-Landau equation. Mathematische Annalen, 328(1):1 $-25,2004$.

[11] M. Jakszto. Another Proof That $l^{p}$-Bounded Pointwise Convergence Implies Weak Convergence. Real Analysis Exchange, 36(2):479 - 482, 2010.

[12] L. Maia, E. Montefusco, and B. Pellacci. Positive solutions for a weakly coupled nonlinear Schrödinger system. Journal of Differential Equations, 229(2):743 - 767, 2006.

[13] R. Mandel. Minimal energy solutions for repulsive nonlinear Schrödinger systems. Journal of Differential Equations, 257(2):450 - 468, 2014.

[14] R. Mandel. Minimal Energy Solutions and Infinitely Many Bifurcating Branches for a Class of Saturated Nonlinear Schrödinger Systems. Advanced Nonlinear Studies, 16(1):95 - 113, 2015.

[15] R. Mandel. Minimal energy solutions for cooperative nonlinear Schrödinger systems. Nonlinear Differential Equations and Applications NoDEA, 22(2):239 - 262, 2015.

[16] R. Mandel, E. Montefusco, and B. Pellacci. Oscillating solutions for nonlinear Helmholtz equations. Zeitschrift für angewandte Mathematik und Physik, (6):121, 2017.

[17] R. T. Rockafellar. Convex Analysis. Princeton University Press, Princeton, New Jersey, 1970.

$[18]$ E. M. Stein. Harmonic analysis: real-variable methods, orthogonality, and oscillatory integrals. Monographs in harmonic analysis 3, Princeton mathematical series, 43. Princeton Univ. Pr., Princeton, NJ, 2nd edition, 1995.

[19] A. Szulkin and T. Weth. Ground state solutions for some indefinite variational problems. Journal of Functional Analysis, 257(12):3802 - 3822, 2009.

R. MANDEL

Karlsruhe Institute of Technology

INSTITUTE FOR ANALYSIS

ENGLERSTRASSE 2

D-76131 Karlsruhe, Germany

E-mail address: rainer.mandel@kit.edu

D. SCHEIDER

Karlsruhe Institute of Technology

INSTITUTE FOR ANALYSIS

ENGLERSTRASSE 2

D-76131 Karlsruhe, Germany

E-mail address: dominic.scheider@kit.edu 